Aus der Abteilung Strahlentherapie und Radioonkologie

(Prof. Dr. rer. nat. Dr. med. C.F. Hess)

im Zentrum Radiologie

der Medizinischen Fakultät der Universität Göttingen

\title{
Inzidenz von Zweittumoren bei Patienten mit zuvor kurativ behandeltem Tumor im Hals-Nasen-Ohren-Bereich
}

\author{
eine prospektive Analyse
}

\author{
INAUGURAL-DISSERTATION \\ zur Erlangung des Doktorgrades \\ der Medizinischen Fakultät \\ der Georg-August-Universität zu Göttingen \\ vorgelegt von \\ Cornelia Ruth Marie Wolff geb. Vienken \\ aus \\ Trier
}

Göttingen 2012 
Dekan: Prof. Dr. med. C. Frömmel

I. Berichterstatter: Prof. Dr. med. H. Christiansen

II. Berichterstatter/in: Prof. Dr. med. C. Hommerich

III. Berichterstatter/in:

Tag der mündlichen Prüfung: 22. Mai 2012 
Inhaltsverzeichnis

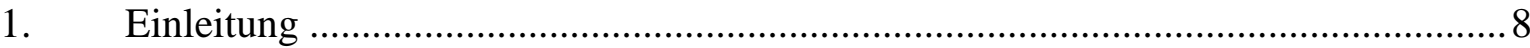

1.1 Inzidenz und Einteilung des Plattenepithelkarzinoms im HNO-Bereich ................8

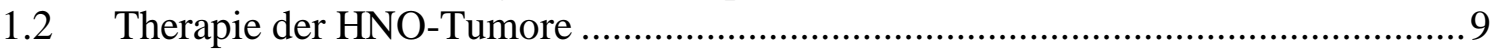

1.3 Risikofaktoren des Plattenepithelkarzinoms im HNO-Bereich ............................. 10

1.4 Epidemiologie und Risikofaktoren bei Bronchialkarzinomen ............................. 11

1.5 Epidemiologie und Risikofaktoren für Ösophaguskarzinome............................... 12

1.6 Gemeinsame Risikofaktoren für HNO-, Bronchial- und Ösophaguskarzinome ... 13

1.7 Zweittumorauftreten bei HNO-Tumorpatienten .............................................. 14

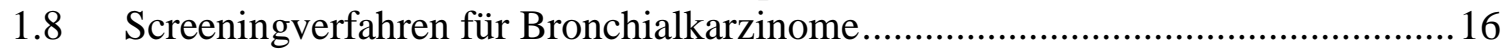

1.9 Screeningverfahren für Zweittumore im Bereich des Ösophagus .......................... 17

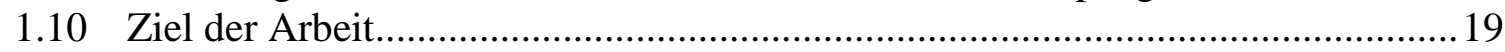

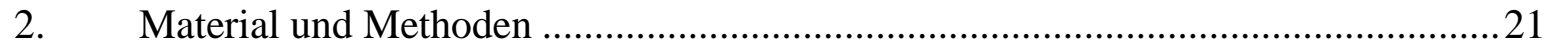

2.1 Eingeschlossene Patienten und Therapie des Primärtumors ................................ 21

2.2 Lokalisation des Primarius sowie TNM- und UICC-Stadien aller

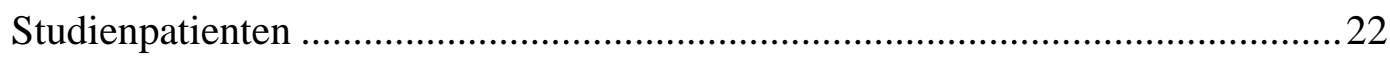

2.3 Patientenrekrutierung............................................................................... 23

2.4 Computertomographie der Lunge und Auswertung der Bildgebung ....................24

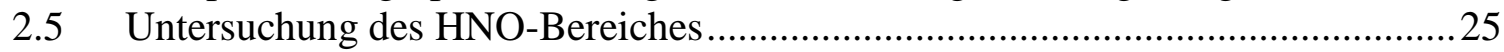

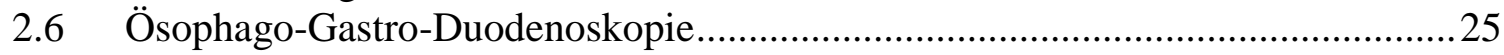

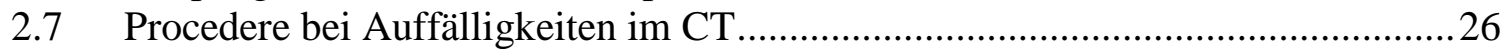

2.8 Procedere bei Auffälligkeiten in der HNO-Untersuchung …...............................27

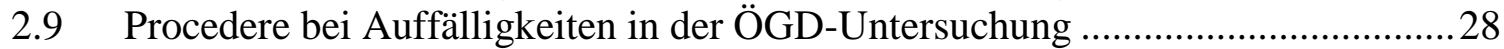

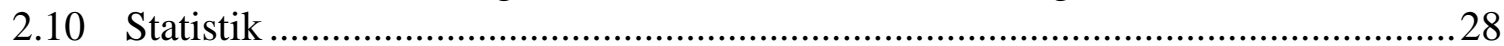

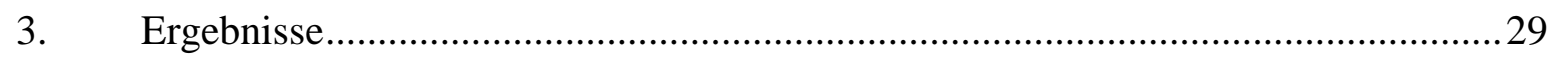

3.1 Durchgeführte Untersuchungen bei allen Studienpatienten ................................29

3.2 Ergebnisse der CT-Thorax-Untersuchungen und weiteres Procedere bei

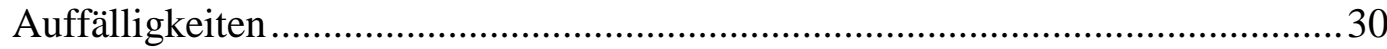

3.2.1 Ergebnisse bei den Patienten, bei denen bereits bei der Primäraufnahme malignomverdächtige Auffälligkeiten beschrieben worden waren .................. 30

3.2.2 Ergebnisse bei Patienten, bei denen Kontrollen in definierten Zeitabständen

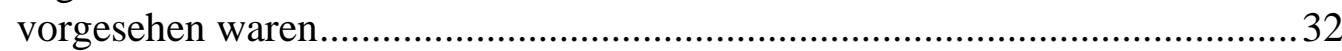

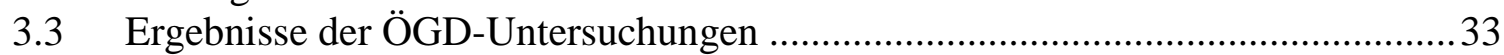

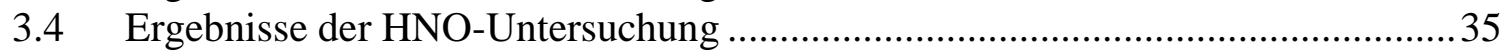

3.5 Zusammenfassungen sämtlicher Untersuchungen bei allen 118 Patienten .............36

3.6 Statistische Wahrscheinlichkeiten eines Zweittumors........................................... 40

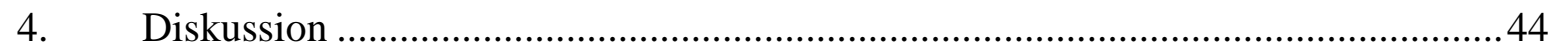

4.1 Inzidenz von Zweittumoren und Screening der Luftwege ..................................4 44

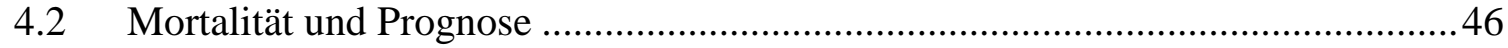

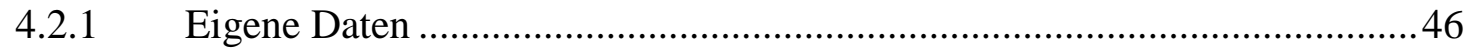

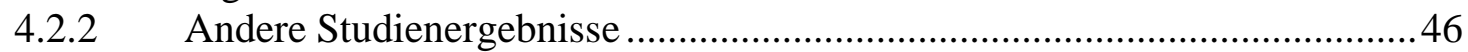

4.2.3 Vergleich mit Screening-Untersuchungen anderer Tumorentitäten............. 49

4.3 Zusammenfassung der vorliegenden Studienergebnisse bezüglich des Benefits eines Computertomographie-gestützten Screenings auf Lungentumore ...............50

4.4 Betrachtung des speziellen Kollektivs dieser Arbeit ..........................................51 
4.5 Screening-Untersuchungen bei HNO-Tumorpatienten 54

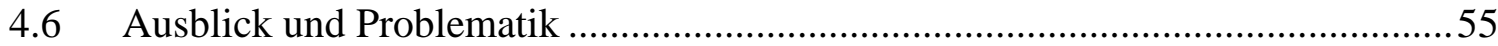

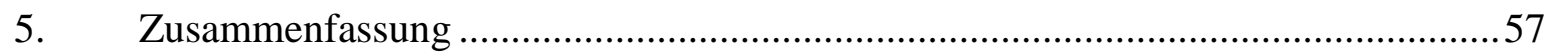

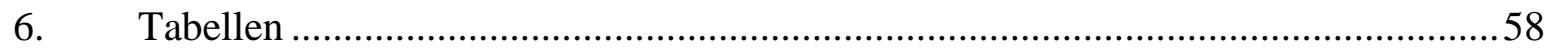

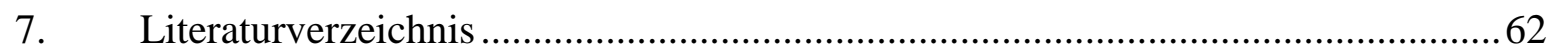




\section{Verzeichnis der Abbildungen}

Abbildung 1: Durchgeführte Untersuchungen aller Studienpatienten aufgeteilt nach Untersuchungsmodalität. ....

Abbildung 2: Ergebnisse der CT-Thorax-Untersuchungen.

Abbildung 3: Ergebnisse der ÖGD-Untersuchungen.

Abbildung 4: Ergebnisse der HNO-Untersuchungen.

Abbildung 5: Zusammenfassung des Ablaufs aller Untersuchungen mit folgender Erläuterung der weiterführenden Untersuchungen bei Malignomverdacht.

Abbildung 6: Malignomverdachte aufgeteilt auf die einzelnen Untersuchungen, sowie Verdeutlichung der positiven Bestätigungsbefunde mit anschließender Therapieintention, was bedeutet, ob eine kurative oder palliative Therapiestrategie verfolgt wurde.

Abbildung 7: Entgültige Gesamtdarstellung der Malignomanzahl plus letztendlicher Therapieintention

Abbildung 8: Darstellung der Gesamtwahrscheinlichkeit eines Zweittumorausbleibens anhand einer Kaplan-Meier-Kurve.

Abbildung 9: Darstellung der Häufigkeiten des Auftretens eines Zweittumors innerhalb eines bestimmten Zeitraums 


\section{Verzeichnis der Tabellen}

Tabelle 1: Verteilung der Lokalisation der Primärtumore, TNM-Stadien- und

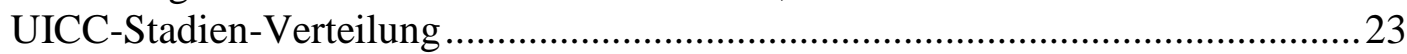

Tabelle 2: Kriterien der Fleischner Society für das Umgehen mit kleinen pulmonalen Herdbefunden im CT bei Risikopatienten 25

Tabelle 3: Zweittumorinzidenz mit Lokalisationsangabe und Therapieintention 40

Tabelle 4: Nikotin- und Alkoholkonsum der Patienten .43

Tabelle 5: T-Stadien der Tumoren von Lippe, Mundhöhle und Oropharynx 58

Tabelle 6: T-Stadien der Tumoren des Hypopharynx 58

Tabelle 7: T-Stadien der Tumoren der Glottis 59

Tabelle 8: T-Stadien der Tumoren der Supraglottis 59

Tabelle 9: T-Stadium der Tumoren der Subglottis 60

Tabelle 10: Stadieneinteilung der Lymphknotenmetastasen bei HNO-Tumoren 60

Tabelle 11: Stadieneinteilung der Fernmetastasen bei HNO-Tumoren 60

Tabelle 12: UICC Stadien 61 


\section{Verzeichnis der Abkürzungen}

ASS:

BWK:

bzw.:

ca.:

CCC:

COPD:

CT:

et al.:

ggf.:

GIST:

Gy:

HNO-Tumor

HP:

MRT:

ÖGD:

PSA:

TNM:

UICC:

UMG:

Vgl.:

z.B.:
Acetylsalicylsäure

Brustwirbelkörper

beziehungsweise

circa

Comprehensive Cancer Centre

chronic obstructive pulmonary disease

Computertomographie

et alii

gegebenenfalls

Gastrointestinaler Strumatumor

Gray

Hals-Nasen-Ohren-Tumor

Helicobacter pylori

Magnetresonanztomographie

Ösophago-Gastro-Duodenoskopie

Prostataspezifisches Antigen

Tumor/Node/Metastasis

Union internationale contre le cancer

Universitätsmedizin Göttingen

Vergleiche

zum Beispiel 


\section{1. $\quad$ Einleitung}

\subsection{Inzidenz und Einteilung des Plattenepithelkarzinoms im HNO-Bereich}

Weltweit sind maligne Neubildungen der Mundhöhle, des Larynx, des Oro- und Hypopharynx mit bis zu 900.000 Neuerkrankungen jährlich (St John et al. 2006) die sechsthäufigste Tumorentität beim Mann (Moral und Paramio 2008). Frauen erkranken seltener, jedoch steigt die Zahl der Neuerkrankungen proportional mit der Anzahl der weiblichen Raucher kontinuierlich an (Strutz und Mann 2001).

So erkrankten allein in Deutschland im Jahr 2006 ca. 12.000 Männer und ca. 3500 Frauen an einem HNO-Tumor (Hentschel und Katalinic 2008). Der Altersgipfel lag dabei beim Mann zwischen dem 50. und 70., bei der Frau zwischen dem 60. und 80. Lebensjahr (Mashberg und Samit 1995).

In Entwicklungsländern ist die Erkrankungsrate dabei deutlich höher als in hoch entwickelten Ländern (Chin et al. 2006), wobei sowohl hier als auch in den Industrieländern Tumore häufiger mit einem niedrigen sozioökonomischen Status verbunden sind (Curado und Hashibe 2009).

Häufigste Lokalisation von HNO-Tumoren ist dabei die Mundhöhle (Simon und Plinkert 2008). Hierzu zählt man den oberen und unteren Alveolarfortsatz, den harten Gaumen, den Zungenrücken vor den Papillae vallatae, die Zungenunterseite sowie den Mundboden (Wittekind und Meyer 2010; Boenninghaus und Lenarz 2005).

Weiterhin treten Tumore auch im Oropharynx mit dem Zungengrund, dem Gaumenbogen mit Tonsillen, sowie der Uvula und der oberen Fläche des weichen Gaumens auf (Wittekind und Meyer 2010; Boenninghaus und Lenarz 2005).

Außerdem kann der Hypopharynx, der in die Postkrikoidgegend, in den Sinus piriformis und die Hypopharynxhinterwand eingeteilt wird (Wittekind und Meyer 2010; Boenninghaus und Lenarz 2005) sowie der Larynx mit seinem supraglottischen, glottischen und subglottischen Raum betroffen sein. Zu supraglottischen Karzinomen zählen dabei Tumore der suprahyoidalen und infrahyoidalen Epiglottis, der aryepiglottischen Falte, der Arythenoidgegend und der Taschenfalten. Karzinome der Glottis können sich an den Stimmlippen, der vorderen und der hinteren Kommissur befinden, der subglottische Bereich wird nicht weiter unterteilt. Als transglottisches Karzinom bezeichnet man ein Karzinom, welches alle drei Teilbereiche befällt. 
Die häufigste Histologie der soliden HNO-Tumore ist das Plattenepithelkarzinom, welches 90\% aller Fälle ausmacht. Seltener gibt es Adenokarzinome, Sarkome oder lymphoepitheliale Tumore, welche allerdings häufig ein anderes Risikoprofil aufweisen (Riede et al. 2004). Die Einteilung der Tumorstadien erfolgt nach der TNM-Klassifikation (englisch: T=Tumour, $\mathrm{N}=$ Node, M=Metastasis) und nach den UICC-Stadien (Union Internationale Contre le Cancer) (Wittekind und Meyer 2010, siehe Tabelle 5-12).

\subsection{Therapie der HNO-Tumore}

Bei der Therapie unterscheidet man grundsätzlichen einen kurativen und palliativen Ansatz. Um über diesen Therapieansatz eine eindeutige Aussage machen zu können, werden Patienten, bei denen ein Hals-Nasen-Ohren-Tumor festgestellt wurde, einem sogenannten „Staging“ unterzogen. Hierbei wird zunächst die Größe des eigentlichen Tumors mit histologischem Differenzierungsgrad bestimmt. Außerdem wird mit Hilfe einer Sonographie oder Computertomographie des Halses eine Aussage über einen möglichen Lymphknotenbefall und dessen Ausmaß gemacht. Zuletzt werden mit Hilfe bildgebender Verfahren wie eines Röntgen-Thorax und einer Sonographie des Abdomens Fernmetastasen ausgeschlossen.

Nach Abschluss dieser Untersuchungen kann ein letztendliches Therapiekonzept festgelegt und auf den Patienten zugeschnitten werden.

Bei Patienten, bei denen bereits Fernmetastasen aufgetreten sind und eine Heilung deshalb nicht mehr möglich ist, wird eine Therapie mit palliativer Zielsetzung verfolgt, welche eine symptomorientierte Behandlung beinhaltet.

Bei kurativen Ansätzen ist die Tumorausbreitung loko-regionär begrenzt. Ziel der Behandlung ist es, den Tumor und befallene lokoregionäre Lymphknoten durch eine multimodale Therapie vollständig zu entfernen oder zu zerstören. Prinzipiell wird also eine vollständige Heilung des Patienten angestrebt. Hierfür stehen verschiedene Möglichkeiten, die je nach Tumorstadium und seiner lokalen Ausbreitung, aber auch von dem Allgemeinzustand des jeweiligen Patienten abhängen, zur Verfügung:

Im kurativen Setting wird in Europa zunächst bei jedem Patienten eine vollständige chirurgische Entfernung des Tumors angestrebt, sofern eine Operation in kurativer Intention prinzipiell möglich ist. Hierbei wird neben einer Primärtumorexzision häufig zusätzlich eine Neck dissection durchgeführt, sofern es klinische oder bildgebende Hinweise auf einen Lymphknotenbefall gibt oder ein fortgeschrittener Lokalbefund vorliegt. Im Detail gilt die 
alleinige Operation in Europa in den UICC-Stadien I und II (Boenninghaus und Lenarz 2005; Franzen 2007) als ausreichend. Ab dem UICC-Stadium III (T3 und N+) oder bei einer unvollständigen Resektion des Primärtumors sollte eine adjuvante Radiotherapie erfolgen (St John et al. 2006). Im Falle einer adjuvanten Therapie sollte immer eine Radiotherapie mit konkomitanter Chemotherapie angestrebt werden, da sich durch die kombinierte Therapie sowohl die lokale Kontrolle des Tumors als auch das Gesamtüberleben der Patienten nachweislich verbessern lässt (Bernier et al. 2004; Bernier 2008; Cooper et al. 2004).

Lokal fortgeschrittene Stadien wie zum Beispiel UICC III und IV, die von chirurgischer Seite aufgrund von Tumorausdehnung oder Allgemeinzustand des Patienten als inoperabel eingestuft werden, werden im primären Setting kurativ radiochemotherapiert (Pfister et al. 2011).

\subsection{Risikofaktoren des Plattenepithelkarzinoms im HNO-Bereich}

$\mathrm{Zu}$ den wichtigsten Risikofaktoren für das Plattenepithelkarzinom im HNO-Bereich gehört der Nikotin- und Alkoholabusus. Dabei erhöht bereits der Nikotinkonsum das Erkrankungsrisiko um das Vierfache im Vergleich zur Allgemeinbevölkerung. Das Risiko steigt weiter, je länger der Konsum stattgefunden hat und je früher er begonnen wurde. Dabei spielen neben Zigaretten auch Zigarren und Pfeifen eine Rolle, Auswirkungen von Kautabakgenuss werden diskutiert (Merletti et al. 1989).

Bei zusätzlichem Alkoholabusus steigt das Risiko auf das Fünfzehnfache des Risikos der Normalbevölkerung an (Mashberg und Samit 1995). Dabei ist das Risiko bei Alkoholabusus stärker dosisassoziiert als beim Nikotinabusus. Dies bedeutet, dass sich bei einem Alkoholiker und Raucher das Risiko bei einer Verdopplung des Alkoholkonsums stärker erhöht, als bei einer Verdopplung seines Nikotinkonsums (Mashberg et al. 1981).

Zusammenfassend setzen sich ca. 90\% der Erkrankten aus starken Rauchern und 70\% aus Gewohnheitstrinkern zusammen (Franzen 2007).

Zusätzlich können auch chronische mechanische Reizungen zum Beispiel durch Zähne oder Prothesen, sowie häufiges heißes und scharfes Essen das Erkrankungsrisiko erhöhen (Mashberg und Samit 1995; Boenninghaus und Lenarz 2005). Bezüglich der Inzidenzraten sind diese Risikofaktoren im Vergleich zu Alkohol- und Nikotinkonsum aber von eher niedriger Relevanz. Auch beruflich bedingte Noxen, wie chemische Substanzen (z.B. Chrom, Arsen und Nickel) werden als seltenere Risikofaktoren angeführt (Riede et al. 2004; Boenninghaus und Lenarz 2005). 
Innerhalb des HNO-Bereichs kann man bestimmte Regionen eingrenzen, die, durch die genannten Risikofaktoren und die anatomischen Gegebenheiten, mit einem höheren Risiko, ein Plattenepithelkarzinom zu entwickeln, belastet sind, als andere. So gelten als besonders gefährdete anatomische Regionen der vordere Mundboden (50\%), der ventrolaterale Zungenanteil (18\%) und der weiche Gaumen mit Uvula, Zungengrund und Tonsille (36\%) (Mashberg und Samit 1995).

Wie bei vielen anderen Tumorerkrankungen spielt auch bei den HNO-Tumoren die individuelle Immunkompetenz eine Rolle. So entwickeln Patienten mit Erkrankungen wie zum Beispiel AIDS („Acquired Immune Deficiency Syndrome“), bei denen eine Schwächung des Immunsystems besteht, häufiger Tumore im HNO-Bereich als immunkompetente Menschen. Auch entwickeln sich bei ebendiesen Patienten aus Tumorvorstufen, wie zum Beispiel Dysplasien oder Carcinomata in situ, häufiger invasive Karzinome. Im Gegensatz zur normalen Altersverteilung der HNO-Tumorerkrankungen, bei denen das Risiko erst ab dem 40. Lebensjahr signifikant ansteigt, entstehen die Tumore auf dieser Grundlage meist früher und verlaufen aggressiver als bei anderen Patienten (Mashberg und Samit 1995).

Ebenfalls als Risikofaktor besonders für das Nasopharynxkarzinom im HNO-Bereich wird der Epstein-Barr-Virus diskutiert. Eine Infektion mit diesem Virus ist überdurchschnittlich oft mit besonders diesen speziellen Tumoren assoziiert (Cao et al. 2011).

Außerdem steht auch eine Assoziation mit Tumoren im gesamten HNO-Bereich und einer Infektion mit humanen Papillomaviren zur Diskussion (Barwad et al. 2011).

\subsection{Epidemiologie und Risikofaktoren bei Bronchialkarzinomen}

Bei den Bronchialkarzinomen unterscheidet man histologisch zwischen dem kleinzelligen Bronchialkarzinom (ca. $15 \%$ aller Patienten) und dem Nicht-kleinzelligen Bronchialkarziom, wie dem Plattenepithelkarzinom (ca. $40 \%$ aller Bronchialkarzinome), dem Adenokarzinom und dem großzelligen Bronchialkarzinom (ca. 10\% aller Bronchialkarzinome) und sehr seltenen anderen Karzinomarten (Renz-Polster et al. 2008).

Insgesamt haben Bronchialkarzinome im zeitlichen Verlauf eine steigende Inzidenz und sind zurzeit der häufigste Krebs des Mannes und der fünfthäufigste der Frau (Schweisfurth und Kurbjuhn 2004). Sie stellen weltweit den höchsten Mortalitätsgrund dar, wobei nachgewiesen wurde, dass $71 \%$ der Bronchialkarzinom-assoziierten Mortalität durch Tabakkonsum verursacht wird (Weiderpass 2010). 
Somit muss als übergeordneter und größter Risikofaktor der Nikotinkonsum genannt werden, wobei das Rauchen das Risiko für alle Lungenkrebsformen, besonders aber das des Plattenepithelkarzinoms (90\% der Plattenepithelkarzinompatienten sind Raucher) und des kleinzelligen Bronchialkarzinoms erhöht und dabei signifikant mit der steigenden Anzahl der Packungsjahre korreliert. Dieser Zusammenhang besteht dabei nicht so ausgeprägt für das Adenokarzinom, welches häufiger bei Frauen und Patienten ohne spezifische Risikofaktoren auftritt (Schweisfurth und Kurbjuhn 2004).

Weitere bekannte Risikofaktoren sind die Inhalation von anderen potentiell kanzerogenen Stoffen, wie Asbest, polyzyklischen Kohlenwasserstoffen, radioaktiven Stäuben, Silikaten, Arsen, Chron- und Nickeldämpfen, aber auch das Passivrauchen und die steigende Umweltverschmutzung. Zusätzlich spielt auch eine genetische Disposition in Form von einer positiven Familienanamnese eine Rolle, ebenso das Vorliegen verschiedener Grunderkrankungen wie COPD, Lungenfibrose, Lungenparenchymnarben bei Pneumokoniosen oder Vitamin-A-Mangel (Schweisfurth und Kurbjuhn 2004).

Da beim Bronchialkarzinom oft erst spät nicht-spezifische Symptome wie Husten, Dyspnoe oder Hämoptysis auftreten, wird die Diagnose häufig erst in weit fortgeschrittenen klinischen Stadien gestellt. Hieraus und aus der meist aggressiven Tumorbiologie erklären sich die über alle Stadien gemittelte 5-Jahres-Überlebensrate von lediglich $15 \%$ und der durch die hohe Metastasierungsrate, zumindest im Verlauf, häufig palliative Therapieansatz (Mahadevia et al. 2003; Herth und Becker 2001). In den letzten 20 Jahren konnte diese schlechte Prognose nicht maßgeblich verbessert werden.

\subsection{Epidemiologie und Risikofaktoren für Ösophaguskarzinome}

Bei Männern steht das Ösophaguskarzinom mit insgesamt jährlich über 4000 Neuerkrankungen in Deutschland und ca. 3700 Todesfällen auf Platz 9 der jährlichen Krebstodesfälle. Frauen erkranken mit durch das Robert-Koch-Institut geschätzten 1000 Neuerkrankungen pro Jahr in Deutschland seltener (Bollschweiler et al. 2009). Dabei werden 30-40\% der Ösophaguskarzinome erst im metastasierten Stadium diagnostiziert und sind somit bei Diagnosestellung formell nicht mehr heilbar (Bollschweiler et al. 2009).

Grundsätzlich unterscheidet man bei den Ösophaguskarzinomen histologisch zwei Tumorentitäten: das Adenokarzinom und das Plattenepithelkarzinom. Das Plattenepithelkarzinom tritt häufiger auf, als das Adenokarzinom und ist eher im mittleren 
und oberen Drittel des Ösophagus lokalisiert, wobei das Adenokarzinom bevorzugt im unteren Drittel auftritt (Renz-Polster et al. 2008).

Grundsätzlich gelten dabei für das Plattenepithelkarzinom des Ösophagus vergleichbar mit den Hals-Nasen-Ohren-Tumoren Alkohol- und Nikotinkonsum als die zwei wichtigsten Risikofaktoren (Pelucchi et al. 2011, Gao et al. 2011). Weiterhin spielen ein Vitaminmangel und Nitrosamine in der Nahrung, aber auch die Achalasie, Verätzungen der Speiseröhre in der Vorgeschichte und die familiäre Disposition eine Rolle. Ebenfalls diskutiert werden zu heiße oder scharfe Speisen und Infektionen mit dem Humanen Papillomavirus. Als wichtigster Risikofaktor für das Adenokarzinom im distalen Drittel der Speiseröhre gilt der gastroösophageale Reflux, welcher durch den Konsum von Alkohol verstärkt oder ausgelöst werden kann (Offner 1997).

\subsection{Gemeinsame Risikofaktoren für HNO-, Bronchial- und Ösophaguskarzinome}

Als der wichtigste gemeinsame Risikofaktor für alle drei genannten Tumorerkrankungen gilt das Zigaretten-, aber auch Pfeifen- und Zigarrenrauchen. Das Risiko an einem Bronchialkarzinom zu erkranken erhöht sich bei Verdopplung der täglich gerauchten Zigaretten bereits auf das 2-Fache des Risikos der Normalbevölkerung und bei Verdopplung der gerauchten Jahre steigt das Risiko auf das 5- bis 6-Fache (Schweisfurth und Kurbjuhn 2004). Bronchialkarzinome sind die häufigste krebsbedingte Todesursache bei Männern und auch bei Frauen ist sie ansteigend, da immer mehr Frauen rauchen. Tabak ist für $85-87 \%$ aller Bronchialkarzinome verantwortlich (Herth und Becker 2001). Auch das Risiko, Tumore im oberen Gastrointestinaltrakt, besonders in der Speiseröhre, zu entwickeln, wird durch Tabakkonsum deutlich erhöht (Morita et al. 2010).

Ebenso spielt auch der Alkoholkonsum sowohl bei der Entstehung von HNO-Tumoren als auch bei der Entstehung von Ösophaguskarzinomen eine wichtige Rolle.

Das Risiko für die Entwicklung eines Plattenepithelkarzinoms in der Speiseröhre erhöht sich sowohl bei Alkoholikern, als auch bei Rauchern signifikant und beim Abusus von beiden Noxen zusammen um das 18 fache des Risikos der Normalbevölkerung (Franceschi et al. 1990). 


\subsection{Zweittumorauftreten bei HNO-Tumorpatienten}

Aufgrund dieser gemeinsamen Risikofaktoren haben Patienten mit einem HNO-Tumor ein deutlich höheres Risiko, einen Zweittumor in der Lunge oder im oberen Gastrointestinaltrakt zu entwickeln (Morris et al. 2011). Es gibt bereits einige Studien, die das gemeinsame oder sequentielle Auftreten dieser Tumore in eine enge Verbindung setzen und eine eindeutige Assoziation aufgrund der gemeinsamen Risikofaktoren postulieren.

So beschreiben Tepperman und Fitzpatrick bereits 1981 in einer Studie, in der 377 Patienten mit Mundbodenkarzinomen untersucht wurden, dass bei $27 \%$ der Teilnehmer in einem Zeitraum von 17 Jahren insgesamt 123 Zweittumore diagnostiziert wurden. 67\% dieser Zweittumore waren Plattenepithelkarzinome des oberen Gastrointestinaltrakts oder des Respirationstrakts, wobei sich davon wiederum ein Drittel in der Mundhöhle befand (in dieser Studie wurde die Mundhöhle zum Gastrointestinaltrakt gerechnet). Diese Tumore entwickelten sich in einer konstanten Rate von 3,6\% pro Jahr und führten zu einer Mortalität von 5,2\% pro Jahr. Abschließend postulierten die Autoren eine engmaschige Kontrolle für dieses Risikopatientenkollektiv (Tepperman und Fitzpatrick 1981).

Heutzutage kann man sagen, dass nicht nur bei Mundbodenkarzinomen, sondern auch bei Tumoren im gesamten HNO-Bereich anhaltender Alkohol- und Nikotinabusus bei bis zu einem Drittel der Patienten zu Zweittumoren führt (Bradley und Bradley 2010). Die jährliche Rate für das Entstehen eines Zweittumors liegt zwischen 6\% und 9\% (Bradley und Bradley 2010).

Dass dabei die gleichen Risikofaktoren Einfluss auf verschiedene Systeme haben können, ist schon länger bekannt.

Slaughter et al. verwendeten bereits 1953 in einer Theorie den Begriff „field cancerization“, um den hohen Prozentsatz an Zweittumoren bei umweltinduzierten Primärtumoren zu unterstreichen (Slaughter et al. 1953).

Die Assoziation mit den genannten Risikofaktoren wird auch dann deutlich, wenn man beachtet, dass Patienten mit Nasopharynxkarzinomen, die zwar auch HNO-Tumore sind, aber nach heutigem Kenntnisstand häufig virusassoziiert und nicht unmittelbar nikotin- und alkoholabhängig entstehen, deutlich weniger Zweittumore entwickeln, als Patienten mit anderen HNO-Tumoren (Tsou et al. 2007). Ebenfalls bemerkenswert ist, dass es auch umgekehrt Daten zum gehäuften Auftreten von HNO-Tumoren bei Ösophaguskarzinompatienten gibt (Watanabe et al. 2007). 
Da die Behandlung der HNO-Tumore in den letzten Jahren immer erfolgreicher geworden ist, werden für diese Patienten zunehmend Zweittumore lebenslimitierend. So bedeutet das Auftreten eines Zweittumors generell eine Verschlechterung der Gesamtprognose, insbesondere wenn er außerhalb des Gebietes des Ersttumors liegt (Bradley und Bradley 2010). Bis zu 82\% der Patienten mit einem Zweittumor stellen sich erst mit Symptomen oder klinischen Zeichen vor, sodass der Tumor häufig schon in einem fortgeschrittenen Stadium ist. Die Patienten sind trotz Symptomen selten dazu motiviert, frühzeitig zum Arzt zu gehen (Dhooge et al. 1998).

Grundsätzlich kann man zwei verschiedene Arten von Zweittumoren unterscheiden. Es gibt Zweittumore, die sich innerhalb von 6 Monaten nach Diagnose des Ersttumors manifestieren. Diese Zweittumore nennt man synchron. Zur Festlegung, dass es sich hier um einen Zweittumor handelt, muss zwischen den beiden malignen Tumoren mindestens $2 \mathrm{~cm}$ gesundes Gewebe vorhanden sein. Weiterhin unterscheidet man den metachronen Zweittumor, welcher impliziert, dass zwischen dem Auftreten des Primärtumors und dem Zweittumor mehr als 6 Monate liegen (Bradley und Bradley 2010).

Aufgrund der besseren Therapien und Lebenserwartungen ist heute nicht nur das Risiko für die Entwicklung von Zweittumoren von Bedeutung, sondern auch das Auftreten von Drittund Vierttumoren. So wurde z. B. in einer Arbeit von Léon et al. 2010 eine retrospektive Analyse mit klinischen Daten von 3631 Patientendaten durchgeführt, die zuvor aufgrund eines HNO-Tumors behandelt wurden. Hier wurde nicht nur das Risiko für die Entwicklung eines Zweittumors untersucht, sondern es wurden auch Risiken für Dritt- und Vierttumore analysiert. Bei der Evaluation stellte sich heraus, dass das Risiko kontinuierlich anstieg. So lag das jährliche Risiko, einen Zweittumor zu entwickeln, bei 3,8\%. Dieses Risiko scheint konstant zu bleiben. Das Risiko für einen Dritttumor, der entsprechend erst nach der Diagnose des Zweittumors auffällt, liegt dann bereits bei 5,1\%. Noch höher ist das Risiko einen Vierttumor zu entwickeln. Dieses liegt dann schon bei 7,8\%. Damit ließen sich signifikante Unterschiede im Risiko der Entwicklung eines Zweit-, Dritt- oder Vierttumors darstellen. Insgesamt fanden die Autoren ein ansteigendes Risiko für Patienten mit vorherigen HNOTumoren, im Verlauf weitere Tumore zu entwickeln (Léon et al. 2010).

Zusammenfassend stellt sich die Frage, ob sich durch ein Screeningverfahren die ÜberlebensRate signifikant verbessern lässt. Besonders Früherkennungsmethoden für Tumore der Lunge werden in der aktuellen Literatur sehr häufig diskutiert. In vielen Studien wird im Rahmen von Screeninguntersuchungen eine Spiral-CT-Aufnahme der Lunge einbezogen. 
In diesem Zusammenhang gibt es bereits mehrere Studien, die sich mit der Frage beschäftigen, ob ein Bronchialkarzinom-Screening mit hochauflösenden Thorax-CTs sinnvoll ist (Mahadevia et al. 2003, Henschke et al. 2001, Herth und Becker 2001, Aberle et al. 2011a, Aberle et al. 2011b). Dies gilt insbesondere für Hoch-Risiko-Patienten, wie ältere, langjährige Raucher, vor allem da man weiß, dass ,in situ“ Bronchial-Karzinome oder mikroinvasive Karzinome im Respirationstrakt eine Heilungschance von nahezu 100\% haben (Herth und Becker 2001).

Weniger dicht ist die Studienlage bei Vorsorgeuntersuchungen der Speisewege in Zusammenhang mit den genannten Risikofaktoren.

Aber auch hier wurde der Benefit von regelmäßigen endoskopischen Screeninguntersuchungen für ösophageale Zweittumore ebenso besonders bei Risikogruppen schon mehrfach geprüft (Petit et al. 2001, Lee et al. 2009).

\subsection{Screeningverfahren für Bronchialkarzinome}

In der Vergangenheit wurden bereits mehrere Studien durchgeführt, die den Nutzen eines möglichen Screeningverfahrens zur früheren Detektion von Bronchialkarzinomen evaluiert haben. Hier ist besonders die Arbeit des „National Lung Screening Trial (NLST)“ zu erwähnen.

Diese Arbeitsgruppe beschäftigt sich intensiv mit dem Benefit eines Lungen-Screenings anhand einer Computertomographie im Vergleich zu einer konventionellen Röntgen-ThoraxAufnahme (Aberle et al. 2011a, Aberle et al. 2011b).

In einer ihrer aktuellsten Arbeiten, veröffentlich im New England Journal of Medicine im April 2011 (Aberle et al. 2011b), schlussfolgert sie eine verminderte Mortalität in Folge eines Bronchialkarzinoms durch eine Computertomographie-Screeninguntersuchung des Thorax.

Um dies zu zeigen wurden von 2002 bis 200453.454 Personen mit hohem Risiko, einen Lungentumor zu entwickeln, randomisiert aufgeteilt in 2 Gruppen. Hohes Risiko bedeutete Personen im Alter zwischen 55 und 74 Jahren mit einer Tabakvorgeschichte von mindestens 30 Packungsjahren. Ehemalige Raucher wurden eingeschlossen, wenn sie innerhalb der letzten 15 Jahre den Konsum eingestellt hatten. Ausgenommen wurden Personen, die vor kurzem die Diagnose eines Lungentumors erhalten haben, innerhalb der letzten 18 Monate eine CT-Untersuchung des Thorax erhalten haben, mit Hämoptysis aufgefallen sind oder einen ungeklärten Gewichtsverlust von mehr als 6,8 kg im letzten Jahr aufwiesen. 
Die eine Gruppe erhielt jährlich über 3 Jahre ein Thorax-CT, die andere eine konventionelle Röntgen-Aufnahme des Brustkorbs. Die relative Reduktion der Mortalität aufgrund eines Lungentumors bei einem CT-Screening der Lunge betrug 20,0\% ( $p=0,004)$. Die Todesrate aufgrund jeglicher anderer Erkrankungen war bei der Gruppe, die die Computertomographie erhielt, im Vergleich zur Röntgen-Thorax-Gruppe um 6,7\% ( $\mathrm{p}=0,02)$ erniedrigt (Aberle et al. 2011b).

In einer anderen Studie von Mahadevia et al. 2003 erhielten in einer Modellstudie mit Hilfe einer Computersimulation 100.000 hypothetische 60-jährige Raucher mit mindestens 20 Packungsjahren, aber in gutem Allgemeinzustand, jährlich eine Spiral-CT-Bildgebung des Thorax. Sie wurden mit einer Gruppe verglichen, die keine CT-Untersuchungen erhielt.

Das Kollektiv wurde nochmals in drei Untergruppen unterteilt, die entweder das Rauchen weiter fortsetzten, zum Zeitpunkt des Screenings mit dem Rauchen aufhörten oder schon seit 5 Jahren aufgehört hatten. Bei diesen Gruppen wurde ein Screening über 20 Jahre simuliert. Gemessen wurden die Vorteile des Screenings, indem der absolute und relative Unterschied des Todeszeitpunktes aufgrund des Lungentumors ermittelt wurde. Das Ergebnis zeigte eine Mortalitätsreduktion von 13\%. Mahadevia et al. legten den Schwerpunkt auf die Kosteneffizienz eines solchen Screenings. Insgesamt ist das Screening nur sinnvoll bei Beginn vor dem Hauptrisikoalter für Bronchialkarzinome, das zwischen dem 67. und 72. Lebensjahr liegt. Zusammenfassend kamen sie zu dem Ergebnis, dass das Screening von Bronchialkarzinomen mit CT-Aufnahmen in Anbetracht der zu niedrigen substanziellen Reduktion der Mortalitätsrate, der vielen falsch positiven Ergebnisse und vor allem der hohen Kosten nicht empfehlenswert ist (Mahadevia et al. 2003).

\subsection{Screeningverfahren für Zweittumore im Bereich des Ösophagus}

Aufgrund des vermuteten erhöhten Risikos für Zweittumore im oberen Gastrointestinaltrakt bei Patienten mit primärem HNO-Tumor und/oder bekanntem Genuss von Tabak und/oder Alkohol, gibt es bereits Veröffentlichungen, die sich mit dem Thema des Zweittumorscreenings für gastro-ösophageale Tumore beschäftigen:

Eine Arbeitsgruppe aus Frankreich hat bei 1560 Patienten mit primärem HNO-Tumor zweimal jährlich über 10 Jahre eine Ösophago-Gastro-Duodenoskopie durchgeführt. Bei 3,2\% wurden metachrone ösophageale Zweittumore entdeckt. Allerdings ist über die Hälfte der Patienten nicht an dem detektierten Zweittumor verstorben und dennoch betrug das 
mittlere Überleben nur 16 Monate, sodass in dieser Arbeit der Benefit eines systematischen Screenings auf ösophageale Zweittumore in Frage gestellt wird (Petit et al. 2001).

Eine weitere asiatische Studie analysierte die Daten des nationalen Krebsregisters Taiwans nach Patienten mit initialem Tumor in Mundhöhle und Pharynx und ösophagealen Zweittumoren bei diesen. Die Analyse lief über 25 Jahre und schloss 33.787 Patienten ein.

Patienten mit Hypopharynxkarzinomen oder Patienten mit Diagnose des Ersttumors im Alter $\leq 50$ Jahre hatten ein erhöhtes Risiko. Allerdings betrug auch hier die Gesamtinzidenz eines Ösophagustumors nur 0,59\%, sodass auch hier das positive Kosten-Nutzen-Verhältnis in Frage gestellt wurde und der Schwerpunkt auf die Detektion bestimmter Hoch-RisikoGruppen gelegt wurde (Lee et al. 2009). 


\subsection{Ziel der Arbeit}

HNO-Tumore spielen weltweit mit 900.000 Neuerkrankungen insbesondere bei Patienten zwischen 50 und 80 Jahren eine wichtige Rolle. Histologisch betrachtet sind die häufigsten Tumore Plattenepithelkarzinome (90\%), gefolgt von seltenen anderen Tumoren.

Es ist bekannt, dass die Entwicklung von Plattenepithelkarzinomen im HNO-Bereich durch bestimmte Risikofaktoren getriggert werden kann. Hier stehen besonders der Tabak- und Alkoholkonsum im Vordergrund. Wobei hier eine eindeutige Assoziation zwischen der Intensität und Dauer des Genusses und dem Auftreten eines Tumors besteht. Besonders bei Kombination dieser beiden Risikofaktoren erhöht sich das Risiko bis auf das 15 fache der Normalbevölkerung.

Betrachtet man andere Tumorentitäten im Zusammenhang mit den Risikofaktoren Tabak und Alkohol, so wurde eindeutig nachgewiesen, dass auch im Bereich des Respirations- und Gastrointestinaltrakts ein höheres Risiko für das Auftreten von Tumoren besteht.

So sind nachgewiesenermaßen $71 \%$ aller Bronchialkarzinom-assoziierten Todesfälle auf Tabakkonsum zurückzuführen. Beim häufigsten Typ der Bronchialkarzinome, dem Plattenepithelkarzinom (40\%), treten sogar 90\% der Tumore bei Rauchern auf.

Im Gastrointestinaltrakt ist besonders der Ösophagus betroffen. So stellen Ösophaguskarzinome beim Mann die 9.-häufigste krebsbedingte Todesursache dar. Auch hier stehen vor allem beim Plattenepithelkarzinom des Ösophagus die Risikofaktoren Alkohol und Tabak im Vordergrund. Aber auch das Adenokarzinom, das hauptsächlich auf der Grundlage eines gastroösophagealen Refluxes entsteht, kann durch Alkoholkonsum und folglich Verstärkung oder Neuauftreten einer Refluxerkrankung hervorgerufen werden.

Aufgrund der gemeinsamen Risikofaktoren für diese Tumorentitäten ist das Ziel der vorliegenden Arbeit, das Auftreten von Zweittumoren in ebendiesen Bereichen bei Patienten, welche bereits zuvor aufgrund eines HNO-Tumors kurativ behandelt wurden, prospektiv zu untersuchen. Dazu wurden bei 118 HNO-Tumor-Patienten im Rahmen der Routinenachsorge eine Computertomographie des Thorax, bei 99 eine Ösophago-Gastro-Duodenoskopie und bei 112 Patienten eine HNO-Untersuchung durchgeführt. Die Ergebnisse der Untersuchungen werden im Rahmen dieser Arbeit prospektiv in Bezug auf die Inzidenz von Zweittumoren in den untersuchten Bereichen bei diesem ausgewählten Patientenklientel mit besonderem Risikoprofil ausgewertet. Anschließend erfolgt eine Analyse der Behandlungsmöglichkeiten 
eventuell diagnostizierter Zweittumore bezüglich eines kurativen oder palliativen Behandlungskonzeptes.

Diese Fragestellungen wurden bislang in der Literatur hauptsächlich retrospektiv und insbesondere nicht im Hinblick auf ein Risikokollektiv mit Patienten mit vorausgegangenem Tumor im HNO-Bereich bearbeitet. Es stellten sich die Fragen, wie hoch die Prävalenz der anderen Tumore nun speziell in dieser Patientengruppe mit vorausgegangenem Risikofaktorassoziiertem Tumor ist und ob man mit regelmäßigen Kontrolluntersuchungen, wie der Computertomographie der Lunge, der Endoskopie der Speiseröhre und des Magens und regelmäßigen HNO-ärztlichen Kontrollen, Zweittumore früh erkennen und vor allem auch kurativ behandeln kann. 
2. Material und Methoden

2.1 Eingeschlossene Patienten und Therapie des Primärtumors

Das Patientenkollektiv bestand aus 118 Patienten die aufgrund eines Tumors im HNOBereich im Zeitraum von 1996 bis 2010 in der Universitätsmedizin Göttingen (UMG) behandelt wurden. Als Einschlusskriterium wurde ein kuratives Behandlungskonzept mit primärer Operabilität ohne Fernmetastasierung bei einem histologisch gesicherten Plattenepithelkarzinom im HNO-Bereich definiert. Patienten mit anderen Tumorhistologien, wie Nasopahrynxkarzinome, wurden aufgrund eines anderen Risikoprofils wie zum Beispiel Virusassoziation, primär ausgeschlossen.

Im Bezug auf das Alter und das Geschlecht der Studienteilnehmer gab es, ebenso wie bei den Risikofaktoren wie Nikotin und Alkohol, keine Ausschlusskriterien. Die Altersverteilung lag zwischen 38 und 86 Jahren (Median 62 Jahre).

Im Rahmen der Studie wurden 13 Frauen und 105 Männer rekrutiert. Während 34 Patienten mit früheren Tumorstadien durch eine lokal kurative Operation ausreichend therapiert werden konnten, erhielten 84 zusätzlich eine adjuvante Radio(chemo)therapie in der Abteilung für Strahlentherapie und Radioonkologie. Von diesen 84 adjuvant behandelten Patienten wurden 25 mit einer alleinigen Radiotherapie behandelt.

Die aktuellen Leitlinien besagen, dass die Tumorstadien UICC I und II alleinig operiert und UICC-Stadien III und IV zunächst operiert und dann adjuvant mit einer Radio(chemo)therapie behandelt oder bei Inoperabilität alleinig radio(chemo)therapiert werden sollten. Im vorliegenden Patientenkollektiv wurden dennoch 5 Patienten im UICCStadium I und II adjuvant Radio(chemo)therapiert, dies erklärt sich bereits durch das Vorliegen eines Rezidivtumors in diesem Stadium, sodass man sich hier trotzdem aufgrund eines hohen Risikos für ein erneutes Lokalrezidiv zu einer adjuvanten Therapie entschieden hat. Außerdem wurden insgesamt 8 Patienten mit UICC-Stadien III bzw. IV nicht adjuvant behandelt. Hier haben die Patienten die postoperative Radio(chemo)therapie ausdrücklich abgelehnt.

Die adjuvante Radiotherapie bestand aus einer normofraktionierten Bestrahlung bis zu einer Gesamtdosis von 60 bzw. 64 Gray mit einer Einzeldosis von 2 Gray pro Tag an 5 Tagen pro Woche.

Bei 84 Patienten wurden die ehemalige Primärtumorregion, die befallenen Lymphknoten sowie potenziell befallene Gebiete, einschließlich der supraklavikulären Region, in einer 
ersten Phase bis zu einer Gesamtdosis von 50 Gy bestrahlt. Anschließend wurden der Primärtumor und die Lymphknotenregionen mit histopathologisch nachgewiesenem Lymphknotenbefall mit Kapseldurchbruch bei 20 Patienten mit einem Boost bis zu einer Gesamtdosis von 60 Gy bestrahlt. 64 Patienten erhielten statt einem Boost bis zu einer Gesamtdosis von 60 Gy einen Boost bis zu einer Gesamtdosis von 64 Gy. Die maximale Bestrahlungsdosis am Rückenmark über die gesamte Bestrahlungsserie wurde jeweils auf 45 Gy limitiert.

Von den insgesamt 59 Patienten, welche mit einer konkomitanten Chemotherapie behandelt wurden, erhielten 17 eine Chemotherapie mit $20 \mathrm{mg}$ Cisplatin und $600 \mathrm{mg}$ 5-Fluoruracil an den Tagen 1-5 und 29-33 der laufenden Radiatio und 42 Patienten eine konkomitante Chemotherapie mit $6 \mathrm{mg}$ Cisplatin/ $\mathrm{m}^{2}$ Körperoberfläche an jedem Bestrahlungstag (siehe auch Tabelle 1).

\subsection{Lokalisation des Primarius sowie TNM- und UICC-Stadien aller Studienpatienten}

Die Ausdehnung des Primärtumors der 118 Studienpatienten bei Erstdiagnose war folgendermaßen verteilt: Bei 3 Patienten wurde ein Carcinoma in situ diagnostiziert. Bei 26 Patienten wurde ein T1-Stadium, bei 27 Patienten ein T2-Stadium, bei 37 Patienten ein T3Stadium und bei 25 Patienten ein T4-Stadium vorgefunden.

Bezüglich des Lymphknotenbefalls wiesen 51 Patienten ein NO-Stadium, 11 Patienten ein N1Stadium, 49 Patienten ein N2- und 7 Patienten ein N3-Stadium auf. Bei keinem Patienten wurden im Rahmen der Staginguntersuchungen Fernmetastasen oder Hinweise auf einen Zweittumor festgestellt.

Zusammengefasst wurden 3 Patienten als UICC-Stadium 0, 18 Patienten als UICC-Stadium I, 10 Patienten als UICC-Stadium II, 18 Patienten als UICC-Stadium III und 69 Patienten als UICC-Stadium IV eingestuft (Tabelle 1, vgl. Tabellen 6-13).

Bei 24 Patienten befand sich der Primarius im Bereich der Mundhöhle, 34 Patienten hatten einen Primarius im Bereich des Oropharynx. Bei 12 Patienten befand sich der Ersttumor im Bereich des Hypopharynx und bei 48 Patienten war der Primarius im Bereich des Larynx lokalisiert. 
Tabelle 1: Verteilung der Lokalisation der Primärtumore, TNM-Stadien- und UICC-StadienVerteilung

\begin{tabular}{|c|c|c|c|}
\hline & $\begin{array}{l}\text { Gesamtkollektiv } \\
\mathrm{n}=118(\%)\end{array}$ & $\begin{array}{l}\text { Adjuvante Bestrahlung } \\
\mathrm{n}=84(71,2 \%)\end{array}$ & $\begin{array}{l}\text { Alleinige Operation } \\
n=34(28,8 \%)\end{array}$ \\
\hline $\begin{array}{l}\text { Chemotherapie } \\
\text { Ja } \\
\text { Nein }\end{array}$ & $\begin{array}{l}59(50) \\
59(50)\end{array}$ & $\begin{array}{l}59(70,2) \\
25(29,8)\end{array}$ & $\begin{array}{l}0 \\
0\end{array}$ \\
\hline $\begin{array}{l}\text { Lokalisation } \\
\text { Primarius } \\
\text { Mundhöhle } \\
\text { Oropharynx } \\
\text { Hypopharynx } \\
\text { Larynx }\end{array}$ & $\begin{array}{ll}24 & (20,3) \\
34 & (28,8) \\
12 & (10,2) \\
48 & (40,7)\end{array}$ & $\begin{array}{r}20 \\
31 \\
9 \\
24\end{array}$ & $\begin{array}{r}4 \\
3 \\
3 \\
24\end{array}$ \\
\hline $\begin{array}{l}\text { T } \\
\text { Cis } \\
1 \\
2 \\
3 \\
4\end{array}$ & $\begin{array}{rr}3 & (2,5) \\
26 & (22,0) \\
27 & (22,9) \\
37 & (31,4) \\
25 & (21,2)\end{array}$ & $\begin{array}{r}0 \\
10 \\
17 \\
33 \\
24\end{array}$ & $\begin{array}{r}3 \\
16 \\
10 \\
4 \\
1\end{array}$ \\
\hline $\begin{array}{l}\mathbf{N} \\
0 \\
1 \\
2 \\
3\end{array}$ & $\begin{array}{rr}51 & (43,2) \\
11 & (9,3) \\
49 & (41,5) \\
7 & (5,9)\end{array}$ & $\begin{array}{r}22 \\
10 \\
46 \\
6\end{array}$ & $\begin{array}{r}29 \\
1 \\
3 \\
1\end{array}$ \\
\hline $\begin{array}{l}\text { UICC } \\
0 \\
1 \\
2 \\
3 \\
4\end{array}$ & $\begin{array}{rr}3 & (2,5) \\
18 & (15,3) \\
10 & (8,5) \\
18 & (15,3) \\
69 & (58,5)\end{array}$ & $\begin{array}{r}0 \\
3 \\
2 \\
15 \\
64\end{array}$ & $\begin{array}{r}3 \\
15 \\
8 \\
3 \\
5\end{array}$ \\
\hline
\end{tabular}

\subsection{Patientenrekrutierung}

Die Patienten, welche die oben genannten Einschlusskriterien erfüllten, wurden im Rahmen ihrer regelmäßigen Nachsorgeuntersuchungen in der Abteilung für Hals-NasenOhrenheilkunde oder Strahlentherapie und Radioonkologie über eine mögliche Studienteilnahme informiert.

Die durchgeführten Untersuchungen waren dabei im Rahmen der tumorspezifischen Routinenachsorge indiziert. Ziel der Studie war es, die Ergebnisse der durchgeführten Untersuchungen systematisch prospektiv auszuwerten.

Nach ausführlicher Anamnese und klinischer Untersuchung wurde ein Informationsgespräch über die geplanten Untersuchungen und die Hintergründe der Studie durchgeführt. Bei bestehendem Interesse erfolgte neben einer allgemeinen schriftlichen Einwilligung für die 
Studienteilnahme eine zusätzliche detaillierte Aufklärung über die Ösophago-GastroDuodenoskopie. Nach ebenfalls schriftlicher Einwilligung in ebendiese erfolgte direkt anschließend eine Blutabnahme, um die benötigten Gerinnungsparameter aktuell bestimmen zu können.

Nach erfolgter Einwilligung der Patienten wurden die einzelnen Termine für die Computertomographie, die Magenspiegelung und für die Untersuchung im HNO-Bereich kurzfristig vereinbart und sowohl telefonisch als auch schriftlich mitgeteilt. Um den Patienten mehrfache Anreisen zu ersparen, wurden, wann immer möglich, alle Untersuchungen an einem Tag durchgeführt, sodass die Patienten nur einmal erneut in der Universitätsklinik erscheinen mussten.

Patienten, die zu ihren vereinbarten Terminen nicht erschienen, wurden noch einmal kurzfristig telefonisch kontaktiert. Bei weiterem Interesse an der Studienteilnahme wurden bis zu viermal neue Termine vereinbart.

Nach Erhalt der Befunde von den untersuchenden Ärzten der kooperierenden Abteilungen, der Hals-Nasen-Ohrenheilkunde der UMG, der Abteilung für Diagnostische Radiologie der UMG und der Allgemeinchirurgie der UMG, wurden die Patienten telefonisch informiert und je nach Befund weitere diagnostische oder therapeutische Schritte eingeleitet (siehe auch Kapitel 2.7-2.9).

\subsection{Computertomographie der Lunge und Auswertung der Bildgebung}

Die Computertomographie wurde ohne Kontrastmittel mit einem 64-Zeiler-Spiral-CT (120 $\mathrm{kV}, 20 \mathrm{~mA}$, Schichtdicke 0,625) durchgeführt. Die Aufnahme beinhaltete die gesamte Lungenregion vom Zwerchfell bis zur Lungenspitze, wobei die Patienten während der Aufnahme für ca. 30 Sekunden die Luft anhalten mussten.

Anschließend wurden die digitalen Bilder immer von demselben Radiologen mit langjähriger Erfahrung in der Diagnostik von Thorax-Computertomographien befundet und ausgewertet. Zur Auswertung wurden die Kriterien der Fleischner Society für das Umgehen mit kleinen pulmonalen Herdbefunden bei Hoch-Risiko-Patienten verwendet, die 2005 in der Zeitschrift Radiology veröffentlicht wurden (MacMahon et al. 2005, siehe auch Tabelle 2).

Die Bilder wurden jeweils im Standard-Lungenfenster (Breite $1500 \mathrm{HE}$, Level $600 \mathrm{HE}$ ), im Weichteilfenster (Breite 400HE, Level $40 \mathrm{HE}$ ), und im Knochenfenster (Breite $1000 \mathrm{HE}$, Level 200 HE) befundet. Das Vorgehen bei Auffälligkeiten wird in Kapitel 2.7 beschrieben. 
Tabelle 2: Kriterien der Fleischner Society für das Umgehen mit kleinen pulmonalen Herdbefunden im CT bei Risikopatienten (vergleiche Tabelle von MacMahon et al. 2005, S.398)

\begin{tabular}{|c|c|}
\hline Größe des Herdbefundes & Vorgehen bei Hoch-Risiko-Patienten \\
\hline$\leq 4 \mathrm{~mm}$ & Kontrolle in 12 Monaten, bei Befundkonstanz keine weitere \\
& Kontrolle \\
\hline$>4-6 \mathrm{~mm}$ & Kontrolle in 6-12 Monaten, bei Befundkonstanz Re-Kontrolle in \\
$18-24$ Monaten
\end{tabular}

\subsection{Untersuchung des HNO-Bereiches}

Zum Ausschluss eines Rezidivs oder Zweittumors wurden alle Patienten in der Hals-NasenOhren-Klinik lokal untersucht. Hierfür wurde zuerst die gesamte einsehbare Mundhöhle mit Hilfe einer Spiegellampe ausgeleuchtet und begutachtet. Um auch durch Weichteile wie z. B. die Zunge verdeckte Regionen zu untersuchen, wurde ein Metallspatel verwendet, um das Sichtfeld zu vergrößern. Anschließend wurde durch ein starres Endoskop der Larynx mit den Stimmlippen, die Epiglottis und deren Umgebung in einer Lupenansicht dargestellt.

Das Vorgehen bei Auffälligkeiten wird in Kapitel 2.8 ausführlich beschrieben.

\section{6 Ösophago-Gastro-Duodenoskopie}

Die Aufklärung zur Spiegelung, sowie die nötige Blutabnahme mit Bestimmung der Gerinnungsparameter, wurden während des Eingangsgesprächs durchgeführt. Dabei wurde insbesondere auf eine eventuell nötige Probenentnahme bei auffälligen Schleimhautbefunden eingegangen. Falls der Untersuchungstermin aus organisatorischen Gründen länger als 2 Wochen in der Zukunft geplant wurde, baten wir die Patienten sich einige Tage vor dem Eingriff bei ihrem Hausarzt vorzustellen, um die aktuellen Gerinnungsparameter zu erhalten. Bei Einnahme von gerinnungshemmenden Medikamenten, wie z.B. Acetylsalicylsäure, wurden die Patienten darauf hingewiesen, das Medikament nach Rücksprache mit ihrem 
Hausarzt mindestens 7 Tage vor Untersuchung abzusetzen oder intermittierend durch Heparinspritzen zu ersetzen.

In Einzelfällen war das Absetzen aufgrund eines erhöhten Risikos für eine Verschlechterung der Grunderkrankung nicht vertretbar. In diesen Fällen wurde dann zunächst auf eine Probenentnahme während der ersten explorativen Spiegelung verzichtet und die Indikation zur Histologiegewinnung nur bei etwaigen lokalen Auffälligkeiten neu eruiert.

Bei einem Teil der Patienten lag aufgrund stattgehabter Operationen und Radiotherapie ein lokal sehr empfindlicher und schmerzhafter Mundraum vor. Zudem mussten in Einzelfällen dünnere Endoskope verwendet werden, die es zuließen den Speiseröhreneingang zu passieren. Aus diesen Gründen wurde jedem Patienten eine Sedierung während der Untersuchung angeboten. Das zur Untersuchung benutzte Endoskop, dessen Durchmesser individuell an die Gegebenheiten angepasst, dabei aber maximal 1,0 cm dick war, bestand aus einem flexiblen Schlauch, an dessen Ende eine Lichtquelle und eine Kamera angebracht waren.

Diese Kamera überträgt die Bilder auf einen Monitor, die in Echtzeit die Speiseröhre in ihrer kompletten Länge, den Magen und auch ein Teil des Duodenums darstellt. Bei auffälligen Befunden konnte dann zusätzlich eine kleine Greifzange durch den Schlauch eingeführt und eine Probe zur histopathologischen Aufarbeitung entnommen werden. Das Vorgehen bei Auffälligkeiten wird in Kapitel 2.9 ausführlich beschrieben.

\subsection{Procedere bei Auffälligkeiten im CT}

Bei Normalbefunden im CT erhielten die Patienten im weiteren Verlauf wieder ihre normalen regelmäßigen Nachsorgeuntersuchungen. Patienten, bei denen ein hochgradiger Karzinomverdacht aufgrund einer irregulären Konfiguration eines Rundherdes mit Speculae und/oder „Pleura-Tails“ oder einer Rundherdgröße von über $10 \mathrm{~mm}$ vorlag, wurden umgehend in der hausinternen Tumorkonferenz für Raumforderungen im Thoraxbereich vorgestellt und bei Operabilität weiter in die Thoraxchirurgie überwiesen. Dort wurde entweder eine video-assistierte Thorakoskopie (VATS) oder eine Thorakotomie mit Entfernung des Tumors durchgeführt.

Bei Inoperabilität aufgrund der Größe oder Lage des Tumors oder im Falle einer sicheren Metastasierung erhielten die Patienten entweder - im Falle einer histologischer Sicherung eines Bronchialkarzinoms - eine kurative Radiochemotherapie oder - im Falle einer histologisch gesicherten metastasierten Situation - eine palliative Chemotherapie. Bei kleineren, primär nicht malignomverdächtigen Rundherden wurde im Bezug auf die Größe 
eine Follow-up Untersuchung, wie oben bereits beschrieben, empfohlen. Wir führten alle erstmaligen Kontroll-CTs durch, die bis zu 12 Monate nach Erstuntersuchung stattfinden sollten.

Bei im Verlauf gleichbleibendem oder rückläufigem Größenbefund wurden die Patienten bei nunmehr fehlender weiterer Kontrollbedürftigkeit in der routinemäßigen Nachsorge weiterbetreut. Bei Größenprogredienz oder neu aufgetretenen Auffälligkeiten erfolgte erneut eine Vorstellung in der Thoraxkonferenz des Comprehensive Cancer Centers (CCC) der UMG. Bei Veränderungen der Lunge oder des Bronchialsystems, wie z.B. Mukoidimpaktationen oder Pneumonien, wurden weitere diagnostische Schritte wie z.B. eine Bronchoskopie eingeleitet und gegebenenfalls über die Lungenfachklinik des evangelischen Krankenhauses Weende in Lenglern oder den jeweiligen Hausarzt nötige Therapien oder Kontrollen eingeleitet.

Beim Fall unklarer Raumforderungen oder von der physiologischen Anatomie abweichenden Verhältnissen außerhalb des zu untersuchenden Organs, wie z.B. im abdominalen Bereich, der in der durchgeführten CT-Untersuchung meistens nur teilweise mit angeschnitten wurde, wurden zusätzliche bildgebende Verfahren, wie eine Magnetresonanztomographie bei z.B. auffälligem Nebennierenbefund, oder eine Computertomographie des Abdomens, bei Auffälligkeiten im gastrointestinalen Bereich, unterstützend hinzugezogen.

\subsection{Procedere bei Auffälligkeiten in der HNO-Untersuchung}

Bei klinischen Auffälligkeiten wurden die Patienten erneut einbestellt, um während einer Panendoskopie in Vollnarkose eine histologische Probe des Gewebes zu entnehmen und gegebenenfalls bereits Tumorgewebe entfernen zu können. Bei positiver Histologie wurde zusätzlich eine Computertomographie des Halses durchgeführt, um eine Metastasierung in die Halslymphknoten auszuschließen. Zusätzlich wurden ein Röntgen-Thorax und eine Sonographie des Abdomens veranlasst, um eine Fernmetastasierung auszuschließen. Anschließend wurden die zu resezierenden Malignome laserchirurgisch entfernt und wenn nötig eine (Re-) Neck dissection durchgeführt. Bei Inoperabilität wurde eine mögliche primäre perkutane Radio(chemo)therapie oder - falls der Patient in der Vorgeschichte bereits bestrahlt worden war - eine Brachytherapie evaluiert. 
2.9 Procedere bei Auffälligkeiten in der ÖGD-Untersuchung

Bei der Aufklärung zur ÖGD wurden die Patienten bereits über eine mögliche Probenentnahme aufgeklärt, sodass der untersuchende Arzt schon während der Spiegelung die Möglichkeit hatte, eine Gewebeprobe verdächtiger Strukturen zu entnehmen.

Voraussetzung für diese Entnahme waren zuvor bestimmte normwertige Gerinnungsparameter und rechtzeitig abgesetzte Behandlungen mit Thrombozytenaggregationshemmern. Konnte dies zum Untersuchungszeitpunkt nicht gewährleistet werden, eine Probenentnahme aber notwendig war, wurde ein kurzfristiger zweiter Untersuchungstermin vereinbart, für welchen die Medikation rechtzeitig abgesetzt oder umgestellt werden konnte.

Bei positiver Histologie wurden die Patienten in der gastroenterologischen Tumorkonferenz des CCC der UMG vorgestellt, um über die möglichen Therapieoptionen zu entscheiden. Auch hier wurden bei unklaren Befunden bildgebende Verfahren, wie die Computertomographie, unterstützend hinzugezogen.

\subsection{Statistik}

Alle Risikoparameter wurden in Bezug auf ihren Einfluss auf die Wahrscheinlichkeit für das Ausbleiben eines Zweittumors analysiert. Hierfür wurden Kaplan-Meier-Kurven und CoxRegressionsanalysen (Cox proportional hazard regression) verwendet. Das Signifikanzlevel für alle Tests wurde auf $\alpha=5 \%$ festgelegt. Alle Analysen wurden mit der freien Software R (www.r-project.org) durchgeführt. 
3. Ergebnisse

\subsection{Durchgeführte Untersuchungen bei allen Studienpatienten}

Insgesamt wurden 118 Patienten im Zeitraum von Juli 2008 bis Juli 2010 eingeschlossen. Alle Patienten wurden zuvor im Zeitraum von 1996 bis 2010 aufgrund ihres Primärtumors in der Universitätsmedizin Göttingen behandelt. Während der darauf folgenden routinemäßigen Nachsorge wurden bei allen Patienten verschiedene Untersuchungen zum Ausschluss einer Fernmetastasierung sowie eines Zweittumors durchgeführt. Diese beinhalteten eine Computertomographie des Thorax, eine Ösophago-Gastro-Duodenoskopie und eine Spiegelung des Hals-Nasen-Ohren-Bereiches. Standardmäßig wurden für alle Studienpatienten alle 3 Untersuchungen geplant, es bestand aber auch die Möglichkeit, einzelne Untersuchungen abzulehnen. Letztendlich wurden 118 CT-, 112 HNO-, und 99 ÖGD-Untersuchungen durchgeführt (Abbildung 1).

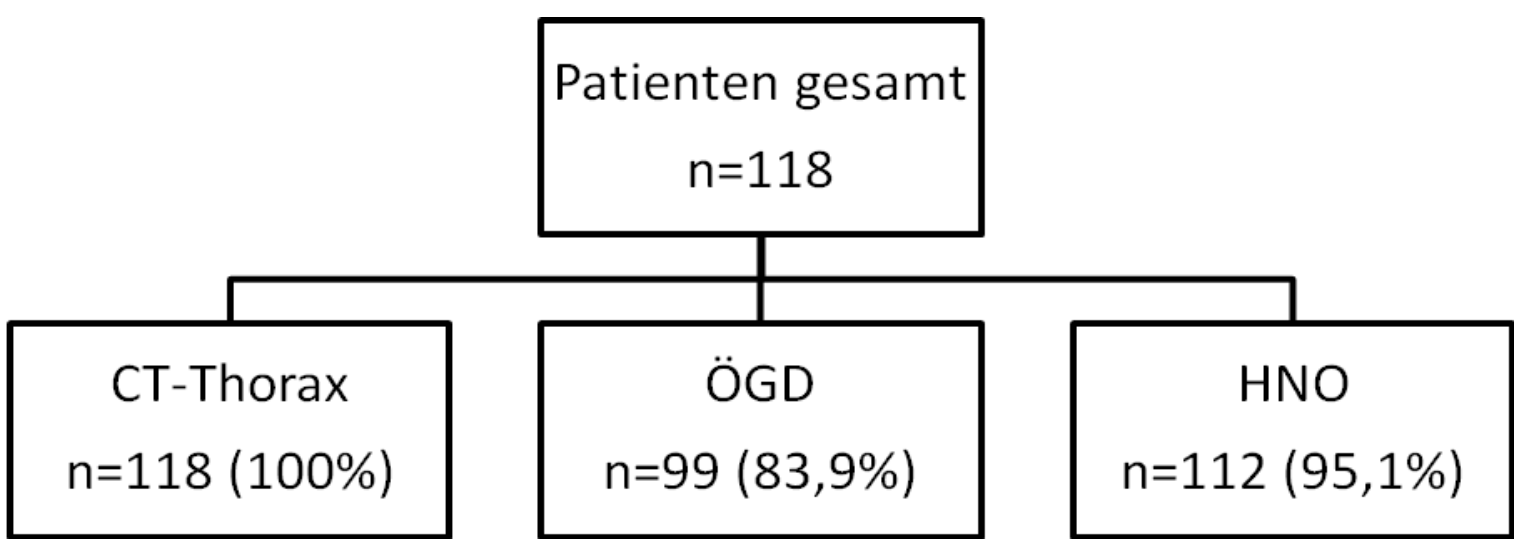

Abbildung 1: Durchgeführte Untersuchungen aller Studienpatienten aufgeteilt nach Untersuchungsmodalität.

$\mathrm{CT}=$ Computertomographie, ÖGD=Ösophago-Gastro-Duodenoskopie, HNO=Hals-NasenOhren, in Klammern=Prozentangaben der Patienten gesamt.

Während demnach bei allen Patienten eine CT durchgeführt werden konnte, lehnten 19 Patienten die geplante ÖGD ab. Als Begründung hierfür wurden hauptsächlich Bedenken, bezüglich des voroperierten und teilweise auch bestrahlten Hals-Nasen-Ohren-Gebietes mit zum Teil starker Vernarbung und Fibrosierung angegeben. Diese Patienten befürchteten, dass 
bei dauerhaft bestehenden Schluckbeschwerden ein Passieren des Endoskops in ebendiesen Bereichen schmerzhaft oder unmöglich sein könnte.

Die angebotene HNO-Untersuchung wurde von 112 der 118 Patienten wahrgenommen. Die restlichen 6 Patienten erschienen trotz ausdrücklicher persönlicher Zusage während des Ambulanzgespräches und mehrmaligen anschließenden Telefonaten nicht zu den vereinbarten Terminen. Hierzu zählten ausschließlich Patienten, die zuvor nach ihrer Operation des Primärtumors adjuvant radiochemotherapiert wurden.

3.2 Ergebnisse der CT-Thorax-Untersuchungen und weiteres Procedere bei Auffälligkeiten

66 der 118 durchgeführten CT-Thorax-Untersuchungen erbrachten Normalbefunde. Diese Patienten konnten anschließend weiter in der Routine-Nachsorge kontrolliert werden. Bei 24 Patienten wurde bereits bei der Primäraufnahme eine Auffälligkeit beschrieben, welche als Malignom eingestuft wurde. Bei diesen 24 Patienten werden im Folgenden dieser Arbeit die Maßnahmen und Ergebnisse der Befundabklärung erläutert, bei weiteren 28 Patienten wurde eine Kontrolle in individuell definierten Zeitabschnitten empfohlen.

\subsubsection{Ergebnisse bei den Patienten, bei denen bereits bei der Primäraufnahme} malignomverdächtige Auffälligkeiten beschrieben worden waren

Bei einem Patienten mit einem bildgebend hochgradigen Verdacht auf ein primäres Bronchialkarzinom mit kontralateraler Lungenmetastasierung wurde eine CT-gesteuerte Punktion durchgeführt, die histologisch ein Plattenepithelkarzinom erbrachte. Aufgrund des vorliegenden Tumorstadiums cT2, cNX, cM1 wurde dieser Patient mit einer palliativen Chemotherapie behandelt.

Außerdem wurden bei 3 anderen Patienten Bronchoskopien durchgeführt, wovon 2 den zuvor geäußerten Verdacht nicht bestätigten und eine ein nicht-kleinzelliges Bronchialkarzinom ergab, welches im Verlauf kurativ operiert werden konnte (pT3 pN0 cM0).

Bei einem weiteren Patienten wurde ein Rundherd reseziert, der sich als Aspergillom herausstellte. 
Bei jeweils 2 Patienten wurden auffällige Nebennierenraumforderungen beschrieben. Hier wurden zur Kontrolle MRTs durchgeführt, die jeweils keine Zweittumoren oder Verdacht auf Metastasen ergaben.

Bei hochgradigem Verdacht auf ein Bronchialkarzinom bei einem weiteren Patienten wurde eine PET-CT-Untersuchung durchgeführt, die den Verdacht bestätigte. Dieser Patient wurde im Verlauf videoassistiert operiert und der Tumor kurativ als primär bronchoalveoläres Adenokarzinom (pT1 cN0 cM0) reseziert.

Bei 4 Patienten wurden Rundherde operativ reseziert. Dabei stellten sich bei 3 Patienten Plattenepithelkarzinome (3mal pT2 pNO cM0) und bei einem ein Adenokarzinom der Lunge (pT1 pN0 cM0) heraus.

Insgesamt bei noch 2 weiteren Patienten wurden Rundherde durch eine videoassoziierte Thorakoskopie kurativ reseziert, diese ergaben zum einen ein Plattenepithelkarzinom (pT2 cN0 cM0) und zum anderen ein adenosquamöses Bronchialkarzinom (pT1 cN0 cM0).

Bei einem weiteren Patienten wurde ein Plattenepithel-Mischtumor (pT4 pN0 cM0) kurativ reseziert.

Bei einem auffälligen Befund des linken Nierensinus wurde ein Patient kurativ an einem Nierenzellkarzinom (pT1a pN0 cM0) operiert.

Bei 2 Patienten wurde ein Verdacht auf ein Ösophaguskarzinom geäußert, welcher sich bei einem Patienten in der ÖGD nicht bestätigte, während der andere durch eine in der ÖGD durchgeführte PE bestätigt werden konnte. Bei diesem Tumor handelte es sich um ein Plattenepithelkarzinom, welches kurativ operiert wurde (pT3 pN0 cM0).

Im Fall einer Raumforderung in der kleinen Magenkurvatur und einer auffälligen ÖGD bei einem Patienten wurden ein CT-Abdomen und Probenentnahmen durchgeführt, die einen gastrointestinalen Strumatumor ergaben, der mit einer Gastrektomie mit D1Lymphadenektomie und einer Chemotherapie mit Imatinib kurativ behandelt wurde.

Ein Patient erhielt bei einem unklaren Lungenbefund zur weiteren Abklärung eine kontrastmittelgestützte CT, welche den Verdacht auf einen Tumor im Bereich der Lunge nicht bestätigte.

Bei einem Patienten wurde bei hochgradigem Verdacht auf ein Bronchialkarzinom und einer Nebennierenraumforderung eine Computertomographie des Abdomens durchgeführt, welche den Verdacht weiterhin bestätigte. Der Patient lehnte allerdings eine VATS als weitere Therapieoption ab. 
In einer anderen CT-Untersuchung eines Patienten fielen ossäre Metastasen der BWK 4-7 des bekannten Oropharynxkarzinoms auf, welche im Verlauf aufgrund von Schmerzen palliativ radiotherapiert wurden.

Eine auffällige Rundherdkonstellation eines weiteren Patienten wurde in der interdisziplinären Tumorkonferenz besprochen und eine Kontroll-CT in 6 Monaten beschlossen. In dieser Kontrolluntersuchung zeigte sich ein Status idem und der Patient konnte weiter in der Routine-Nachsorge kontrolliert werden.

Einem Verdacht auf ein Bronchialkarzinom bei einem Patienten wurde im Verlauf nicht mehr nachgegangen, weil dieser zusätzlich einen inoperablen Tumor im HNO-Bereich aufwies und deswegen palliativ-symptomorientiert behandelt wurde.

3.2.2 Ergebnisse bei Patienten, bei denen Kontrollen in definierten Zeitabständen vorgesehen waren

Bei weiteren 28 Patienten wurde eine Kontrolle in individuell definierten Zeitabschnitten empfohlen. Dieser belief sich, wie bereits in Kapitel 2.4 beschrieben, je nach Befundmorphologie und Größe auf 3, 6 oder 12 Monate nach der Primäraufnahme.

Bei den durchgeführten Kontrollaufnahmen wurden 25 Befunde als unauffällig bzw. unverändert beschrieben, sodass auch diese Patienten weiter in der Routine-Nachsorge kontrolliert werden konnten. Von diesen Patienten wurde einer bei intrapulmonal vergrößerten Lymphknoten innerhalb von 3 Monaten kontrolliert. Bei der Kontrolle waren die Lymphknoten nicht mehr nachzuvollziehen.

13 Patienten erhielten bei kleinen Rundherden von 4-6 mm eine kurzfristige Kontrolle von 6 Monaten, wobei jeweils eine Befundkonstanz oder Regression dokumentiert wurde.

11 Patienten erhielten bei kleinsten Rundherden von $\leq 4 \mathrm{~mm}$ eine Kontrolluntersuchung in 12 Monaten, die alle eine Befundkonstanz zeigten.

Bei 3 Kontroll-Untersuchungen, eine nach 3 Monaten, eine nach 6 und eine nach 9 Monaten, fielen progrediente oder neue Veränderungen auf, welche nun als malignomverdächtig gewertet und einer weiteren Abklärung zugeführt werden mussten.

In einer Kontroll-CT zeigte sich ein hochgradiger Verdacht auf ein Magenkarzinom. Allerdings waren im Verlauf durchgeführte PEs negativ und der Patient lehnte einen operativen Eingriff im Sinne einer Gastrektomie ab.

Einem anderen hochgradigen Verdacht auf ein Bronchialkarzinom in einer Kontroll-CT wurde aufgrund des schlechten Allgemeinzustands des Patienten nicht nachgegangen. 
Zuletzt zeigte sich ein neuer unklarer Rundherd bei einem Patienten, der mit einer atypischen Resektion entfernt wurde und sich als Epitheloidzellgranulom herausstellte.

Zur Zusammenfassung aller durchgeführten Untersuchungen und $\mathrm{zu}$ den bestätigten Malignomen siehe auch Abbildung 5.

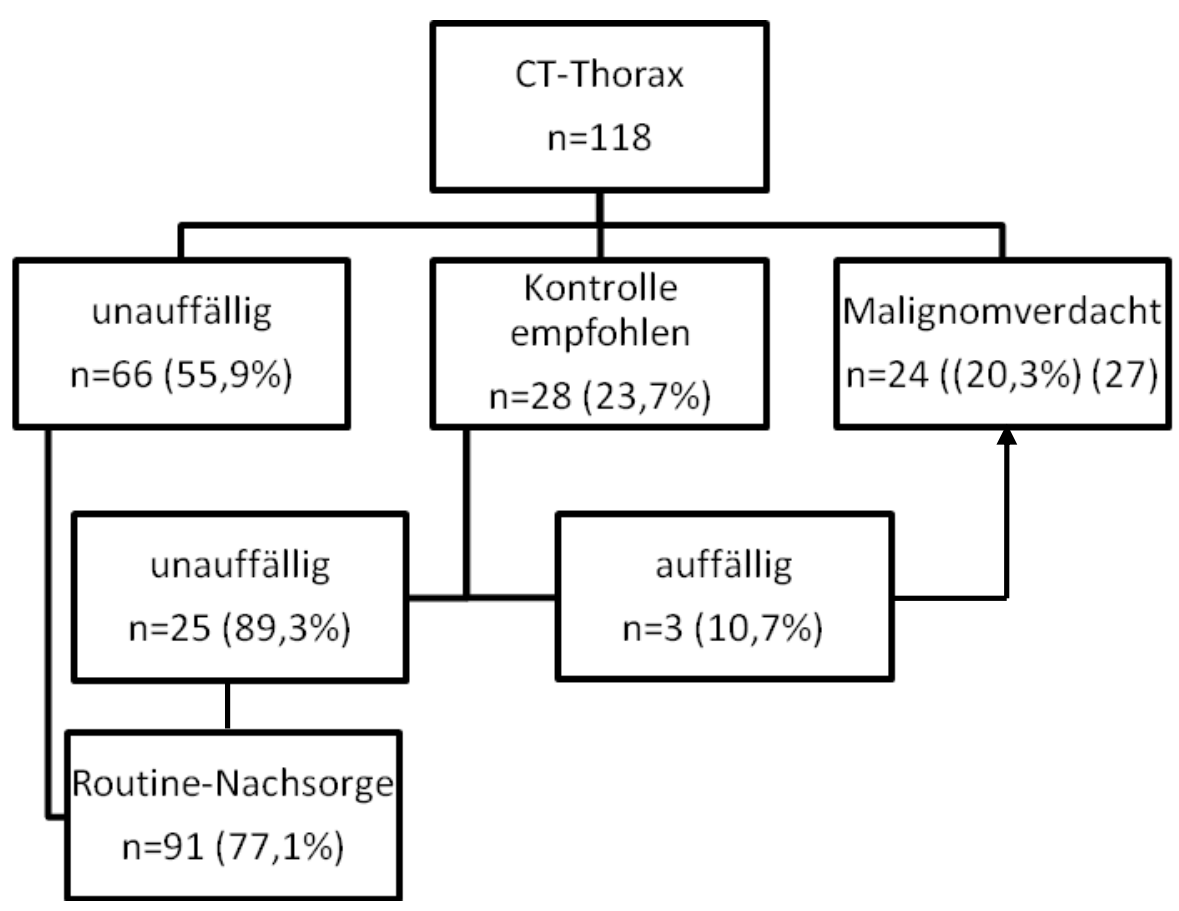

Abbildung 2: Ergebnisse der CT-Thorax-Untersuchungen.

$\mathrm{CT}=$ Computertomographie, in Klammern=Prozent des jeweils übergeordneten Fensters.

\subsection{Ergebnisse der ÖGD-Untersuchungen}

Von 99 Ösophago-Gastro-Duodenoskopien waren 69 (70\%) Untersuchungen primär unauffällig. Diese Patienten werden somit weiter in der Routine-Nachsorge betreut. Bei 20 Patienten (20\%) bestand eine Abweichung vom Normalbefund allerdings ohne weitere akute Kontrollbedürftigkeit oder Malignomverdacht. Dazu gehörten eine Helicobacter-pylori (HP)positive Refluxösophagitis, die mit einer Eradikationstherapie beim Hausarzt behandelt wurde, eine leichtgradige Duodenitis, bei der eine Probeentnahme aufgrund von ASSEinnahme nicht durchgeführt werden konnte, eine leicht unregelmäßige Z-Linie bei ansonsten unauffälligem Befund, dreimal ein Verdacht auf einen Barett-Ösophagus, der sich histologisch nicht bestätigte, ein Ösophagusvarizenbefund Grad I mit hypertensiver Gastropathie, eine inkomplette intestinale Metaplasie bei Refluxösophagitis, ein Befund mit mehreren Ulcera bulbi duodeni, die mit Protonenpumpenhemmern therapiert wurden, drei 
Hernienbefunde, ein Befund mit Magenerosionen, bei denen ebenfalls Protonenpumpenhemmer verschrieben wurden, sowie eine gleichsam behandelte Refluxösophagitis. Außerdem noch ein Verdacht auf eine portal-hypertensive Gastropathie, sowie ein Polypenbefund im Duodenum, mit Empfehlung einer Koloskopie. Weiterhin zweimal ein Ösophagusvarizenbefund mit Begleitgastritis, sowie zweimal leichte Schleimhautentzündungen, die ebenfalls mit Protonenpumpenhemmern behandelt wurden.

Diese Befunde wurden den jeweiligen Hausärzten mitgeteilt, sodass von dort aus regelmäßige Kontrollen bzw. nötige Behandlungen stattfinden konnten. Weiterhin wurden 6 zeitnahe Kontroll-Endoskopien, aufgrund von höhergradigen Schleimhautentzündungen und 2 hypertensiven Gastropathien empfohlen, wovon 3 durchgeführt wurden und einen im Verlauf unauffälligen Befund zeigten. Die anderen 3 Patienten verweigerten auch nach intensiver Aufklärung über mögliche Folgen eine zweite Untersuchung zur Kontrolle.

Bei 4 von 99 Untersuchungen (4\%), ergaben sich Befunde, welche als malignomverdächtig gewertet wurden. Bei 2 dieser Befunde wurde bereits während der endoskopischen Untersuchung eine Probebiopsie durchgeführt. Bei einem weiteren Patienten wurde eine erneute ÖGD mit Probebiopsie und Endosonographie empfohlen. Für den vierten Patienten wurde eine CT-Abdomen-Kontrolle angeschlossen. Insgesamt bestätigten sich 3 der 4 Malignomverdachte histologisch (75\%) (siehe Tabelle 3). Dazu gehörten 2 Plattenepithelkarzinome (pT3 pN0 cM0; ypT0 ypN0 cM0) und ein GIST. Die durchgeführte CT-Abdomen-Untersuchung lieferte hingegen keinen Hinweis auf eine bösartige Erkrankung. Alle 3 bestätigten Malignome wurden durch eine neoadjuvante Therapie mit anschließender Tumorresektion, einer alleinigen Tumorresektion und einer Resektion mit anschließender adjuvanter Therapie (GIST) kurativ behandelt. 


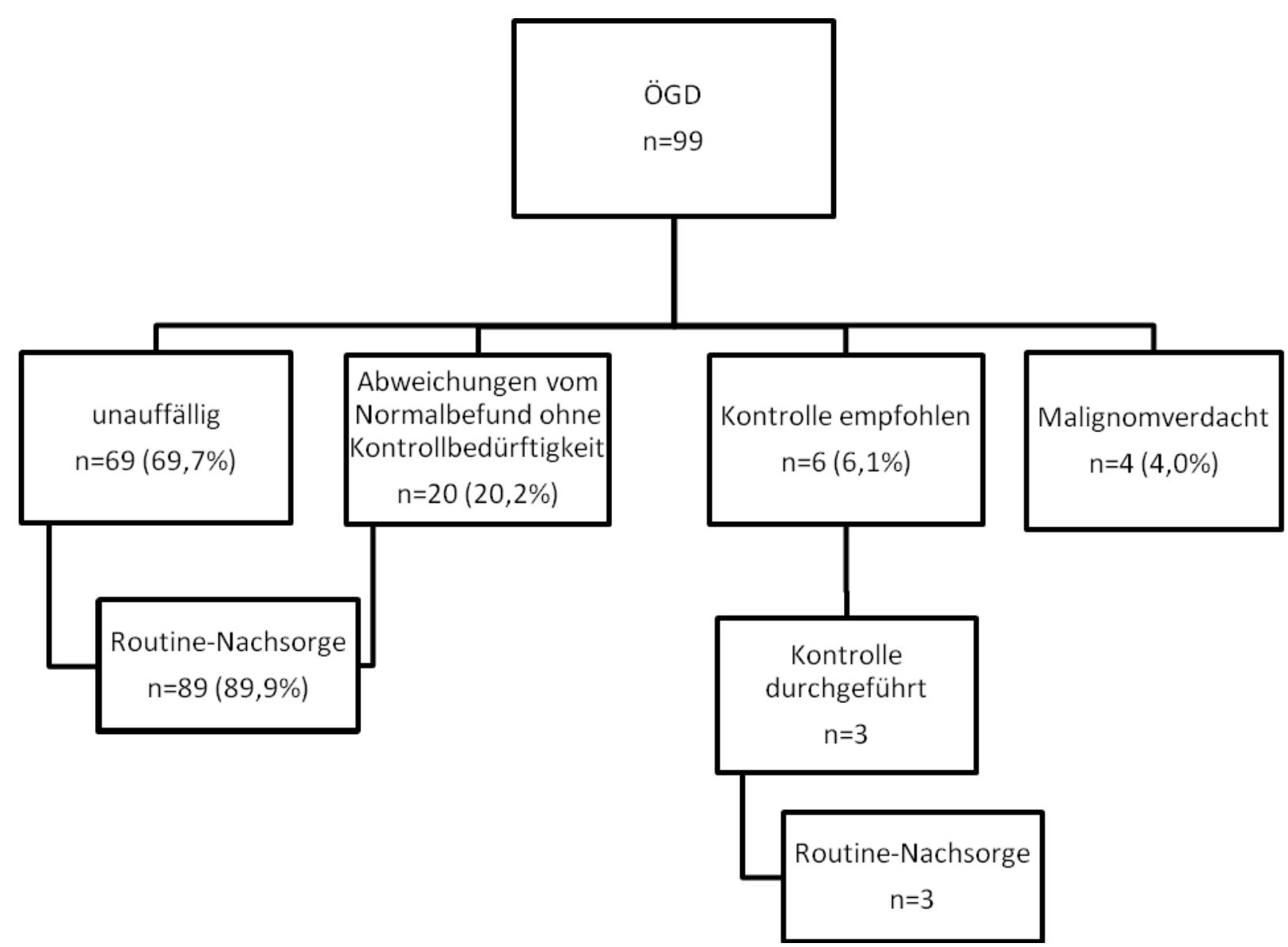

Abbildung 3: Ergebnisse der ÖGD-Untersuchungen.

ÖGD=Ösophago-Gastro-Duodenoskopie, in Klammern=Prozent des jeweils übergeordneten Fensters.

\subsection{Ergebnisse der HNO-Untersuchung}

Von den 112 HNO-ärztlich untersuchten Patienten waren 105 Patienten (94\%) bereits bei der Primäruntersuchung unauffällig und werden somit weiter in der Routine-Nachsorge kontrolliert. Bei 7 Patienten (6\%) bestand der Verdacht auf ein Lokalrezidiv bzw. Zweittumor im Hals-Nasen-Ohren-Bereich. Dieser ließ sich in allen 7 Fällen (100\%) durch Probeentnahmen bestätigen (siehe Tabelle 3).

Bei diesen Tumoren wurde nur ein Tumor, bei einem in dieser Region vorbestrahlten Patienten, als inoperabel eingestuft. Da der Patient eine weitere Chemo- und Brachytherapie ablehnte, wurde hier nur eine palliative Symptomkontrolle verfolgt. Die restlichen 6 Patienten wurden mit einem kurativen Therapieansatz behandelt: bei 2 Patienten wurde allein eine laserchirurgische Resektion als kurative Therapie verwirklicht, ein Patient erhielt eine Brachytherapie bei gleichzeitiger kurativer Therapie seines neuaufgetretenen ÖsophagusKarzinoms, bei 2 Patienten wurde eine Tumorresektion mit Neck dissection und 
anschließender Radiochemotherapie durchgeführt und ein Patient erhielt eine kurative Radiochemotherapie.

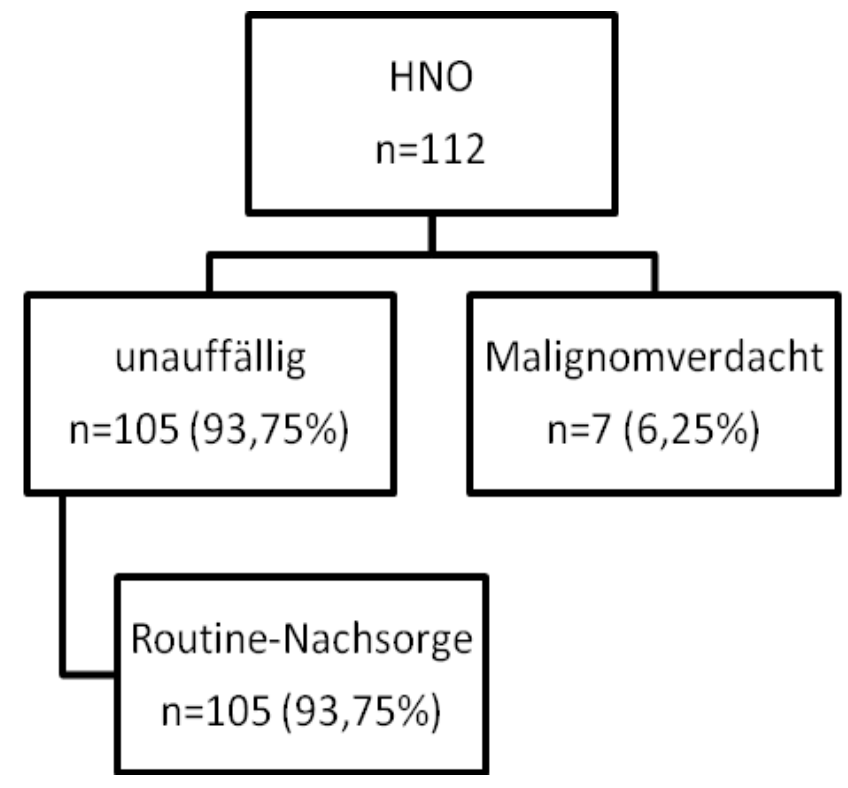

Abbildung 4: Ergebnisse der HNO-Untersuchungen.

$\mathrm{HNO}=$ Hals-Nasen-Ohren, in Klammern=Prozent des jeweils übergeordneten Fensters.

\subsection{Zusammenfassungen sämtlicher Untersuchungen bei allen 118 Patienten}

Von den insgesamt 118 Patienten konnten 59 (50\%) in allen durchgeführten Untersuchungen als unauffällig eingestuft und somit weiter in der normalen Routine-Nachsorge kontrolliert werden. Bei 29 Patienten (25\%) wurde eine Kontroll-Untersuchung empfohlen, wobei 3 Patienten in diesen Zweituntersuchungen einen neuen Malignomverdacht aufwiesen. Diese 3 neuen Malignomverdachtsfälle entstammen allesamt aus Re-CT-Thorax-Untersuchungen.

Bei 30 Patienten (25\%) fiel bereits bei der ersten Untersuchung ein Malignomverdacht auf, sodass zusammenfassend 33 Patienten (28\%) mit einem Verdacht auf ein Malignom weiterführend untersucht und/oder behandelt wurden.

3 der 33 Patienten (9\%) verweigerten aufgrund ihres Allgemeinzustandes weiterführende Maßnahmen. Von diesen 3 Patienten wiesen 2 Patienten (66\%) den Malignomverdacht in den Re-CT-Untersuchungen auf. Bei einem Patienten wurde bereits bei der primären CT-ThoraxUntersuchung ein dringender Verdacht auf ein Bronchialkarzinom ausgesprochen. Die Hausärzte dieser Patienten wurden informiert und es wurde gegenüber den Patienten und ihren Angehörigen, als auch gegenüber den Hausärzten darauf hingewiesen, dass sie jederzeit bei Behandlungsbedarf oder Interesse auf uns zukommen könnten. 
Insgesamt ergeben sich somit 26 bestätigte Malignome in den einzelnen Untersuchungen. In der endgültigen Zusammenfassung werden aber nur 21 Malignome beschrieben. da bei einem Patienten in allen 3 Untersuchungen ein Malignomverdacht geäußert wurde und sich endgültig 2 Malignome bei ebendiesem Patienten bestätigen ließen. Bei der abschließenden Ergebnisanalyse wird dieser Patient einzeln gewertet, da in der Auswertung die einzelne Person und nicht die Anzahl der Tumore zählt. Außerdem wurde bei einem Patienten in der CT- und in der HNO-Untersuchung ein Verdacht auf dasselbe Malignom und bei 2 Patienten in CT- und ÖGD-Untersuchung der Verdacht auf dieselben Malignome geäußert. Somit ergibt sich eine Gesamtzahl von 21 Patienten mit histologisch bestätigten Malignomen (siehe auch Tabelle 3). Von diesen waren zusammengefasst 10 Bronchialkarzinome, 3 gastrointestinale Tumore (2 Ösophaguskarzinome und 1 GIST), 7 Zweittumore im HNO-Bereich, 1 Nierenzellkarzinom und 1 ossäre Metastasierung. 18 Tumore konnten kurativ behandelt werden. Auch hier ist wieder zu beachten, dass ein Patient mit einem lokalen HNO-Tumor und einem Ösophagustumor aufgefallen ist und ebenfalls kurativ behandelt wurde. Bei den kurativ behandelten Tumoren handelte es sich somit zusammenfassend um ein NichtKleinzelliges Bronchialkarzinom ( Adenokarzinom (pT1 cN0 cM0) und ein Adenokarzinom der Lunge (pT1 pN0 cM0), zwei Plattenepithelkarzinome des Ösophagus (pT3 pN0 cM0; ypT0 ypN0 cM0), vier Plattenepithelkarzinome der Lunge (4mal pT2 c/pN0 cM0), ein adenosquamöses Bronchialkarzinom (pT1 cN0 cM0), ein Carcinoma in situ des Stimmbands, ein Nierenzellkarzinom (pT2 pN0 cM0), ein Oropharynxkarzinom (pT2 pN0 cM0), ein OroHypopharynx-Karzinom (pT3 pN0 cM0), ein Gastrointestinaler-Strumatumor, ein Plattenepithel-Mischtumor der Lunge (pT4 pN0 cM0), ein Karzinom im Pharynxschlauch (pT2 cN0 cM0) und ein Larynxkarzinom (pT1 cN0 cM0).

Die palliativ behandelten Tumore bestanden aus einem ausgedehnten inoperablen Lokalrezidiv, welches nach Absprache mit dem Patienten auch nicht mehr mit einer palliativen Brachytherapie, sondern nur noch symptomkontrolliert behandelt wurde, desweiteren aus einer ossären Metastasierung in BWK 4 bis 7 und einem inoperablen Plattenepithelkarzinom der Lunge, welche jeweils palliativ bestrahlt wurden. Eine Analyse bezüglich der Auftrittswahrscheinlichkeit von Zweittumoren bei Patienten mit höheren Primärtumorstadien (UICC III und IV) im Vergleich zu Patienten mit niedrigeren Stadien (UICC I und II) erbrachte keinen signifikanten Unterschied.

Die Zusammenfassungen aller Untersuchungen werden in den Abbildungen 5-7 zusammengefasst. 


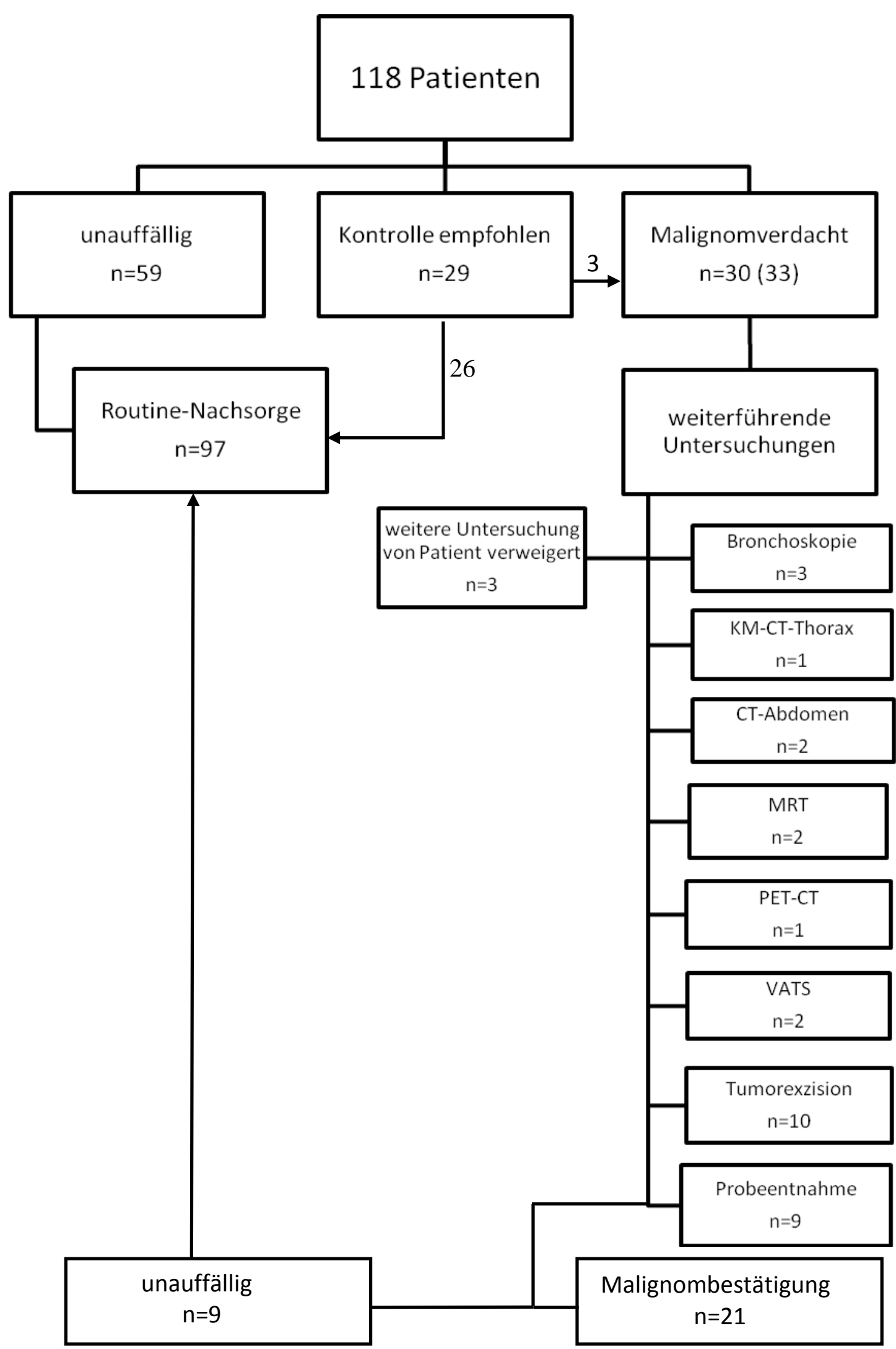

Abbildung 5: Zusammenfassung des Ablaufs aller Untersuchungen mit folgender Erläuterung der weiterführenden Untersuchungen bei Malignomverdacht.

KM-CT=Kontrastmittel-Computertomographie, MRT=Magnetresonanztomographie, PET$\mathrm{CT}=$ Positronen-Emissions-Computertomographie, VATS=video-assistierte Thorakoskopie 


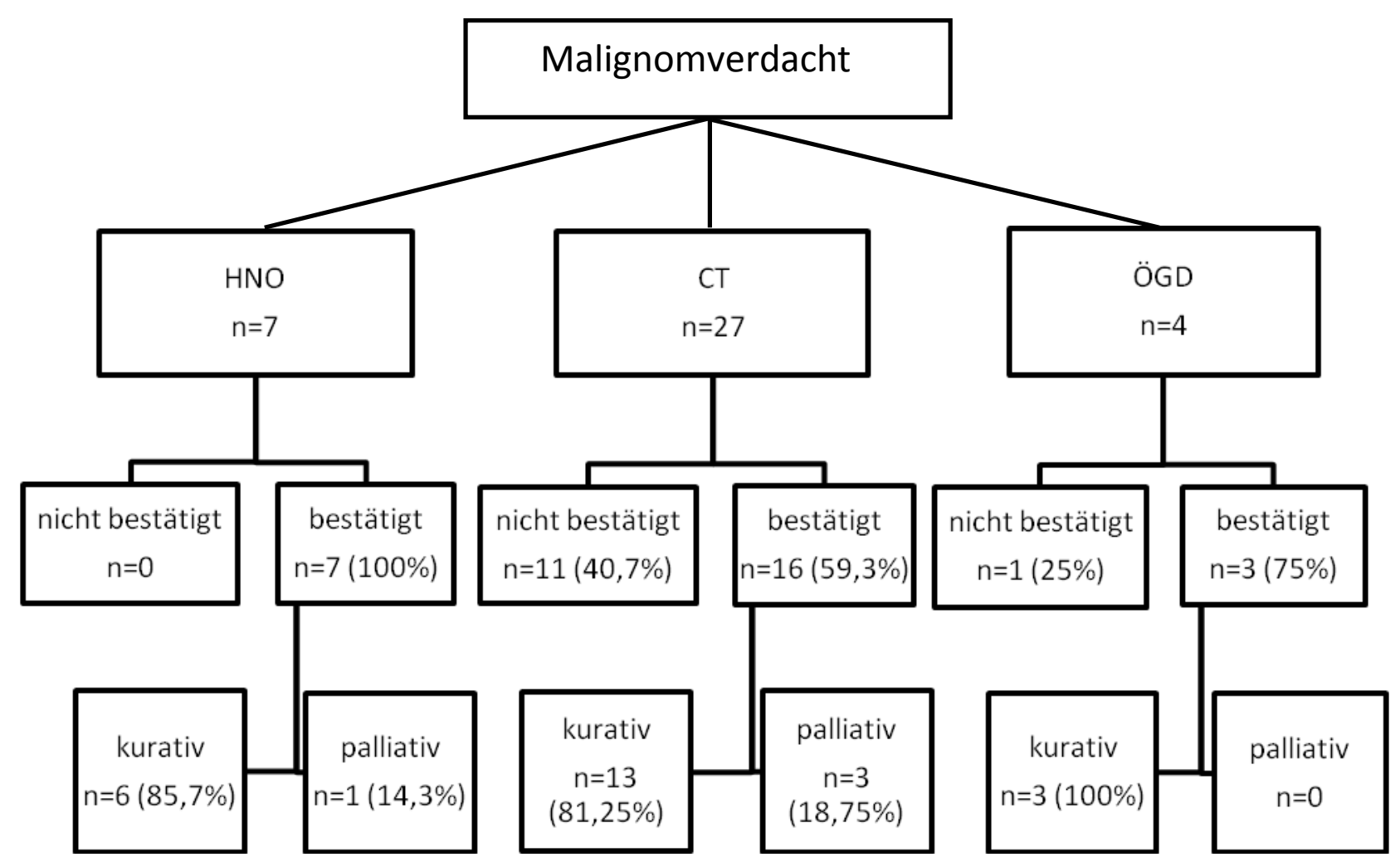

Abbildung 6: Malignomverdachtsfälle aufgeteilt auf die einzelnen Untersuchungen, sowie Verdeutlichung der positiven Bestätigungsbefunde mit anschließender Therapieintention, was bedeutet, ob eine kurative oder palliative Therapiestrategie verfolgt wurde.

$\mathrm{CT}=$ Computertomographie, $\mathrm{HNO}=$ Hals-Nasen-Ohren, ÖGD=Ösophagogastroduodenoskopie, in Klammern=Prozent des jeweils übergeordneten Fensters

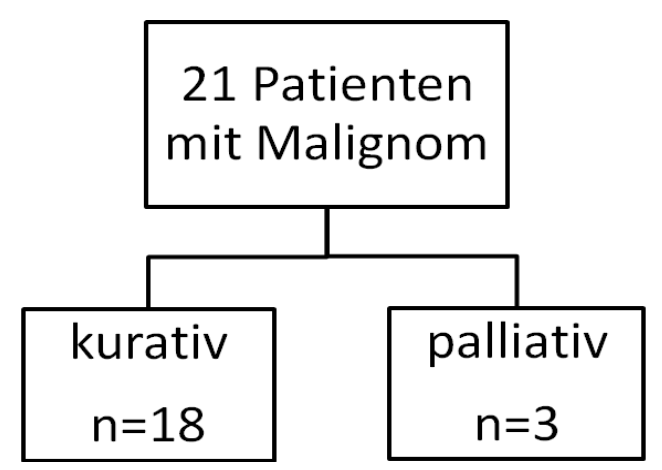

Abbildung 7: Entgültige Gesamtdarstellung der Malignomanzahl plus letztendlicher Therapieintention 
Tabelle 3: Zweittumorinzidenz mit Lokalisationsangabe und Therapieintention

\begin{tabular}{|l|r|}
\hline & $\begin{array}{l}\text { Gesamtkollektiv } \\
\text { n=118(\%) }\end{array}$ \\
\hline Zweittumor & $21(17,8)$ \\
Ja & $97(82,2)$ \\
Nein & \\
\hline Lokalisation & \\
Zweittumor & $7(33,3 \%)$ \\
Lokal & $10(47,6 \%)$ \\
Lunge & $3(14,3 \%)$ \\
GIT & $2(9,5 \%)$ \\
andere & \\
\hline Kurative & \\
Therapie & $18(85,7)$ \\
Ja & $3(14,3)$ \\
nein & \\
\hline
\end{tabular}

\subsection{Statistische Wahrscheinlichkeiten eines Zweittumors}

In der statistischen Auswertung konnte aus den vorher beschriebenen Daten eine KaplanMeier-Kurve errechnet werden, welche die Wahrscheinlichkeit eines Zweittumorereignisses darstellt. Die Ergebnisse zeigen, dass nach 50 Monaten bereits über 20\% der Patienten einen Zweittumor entwickelt haben und nach circa 130 Monaten, also nach knapp 11 Jahren, 40\% der Patienten einen Zweittumor aufwiesen (Abbildung 8). 


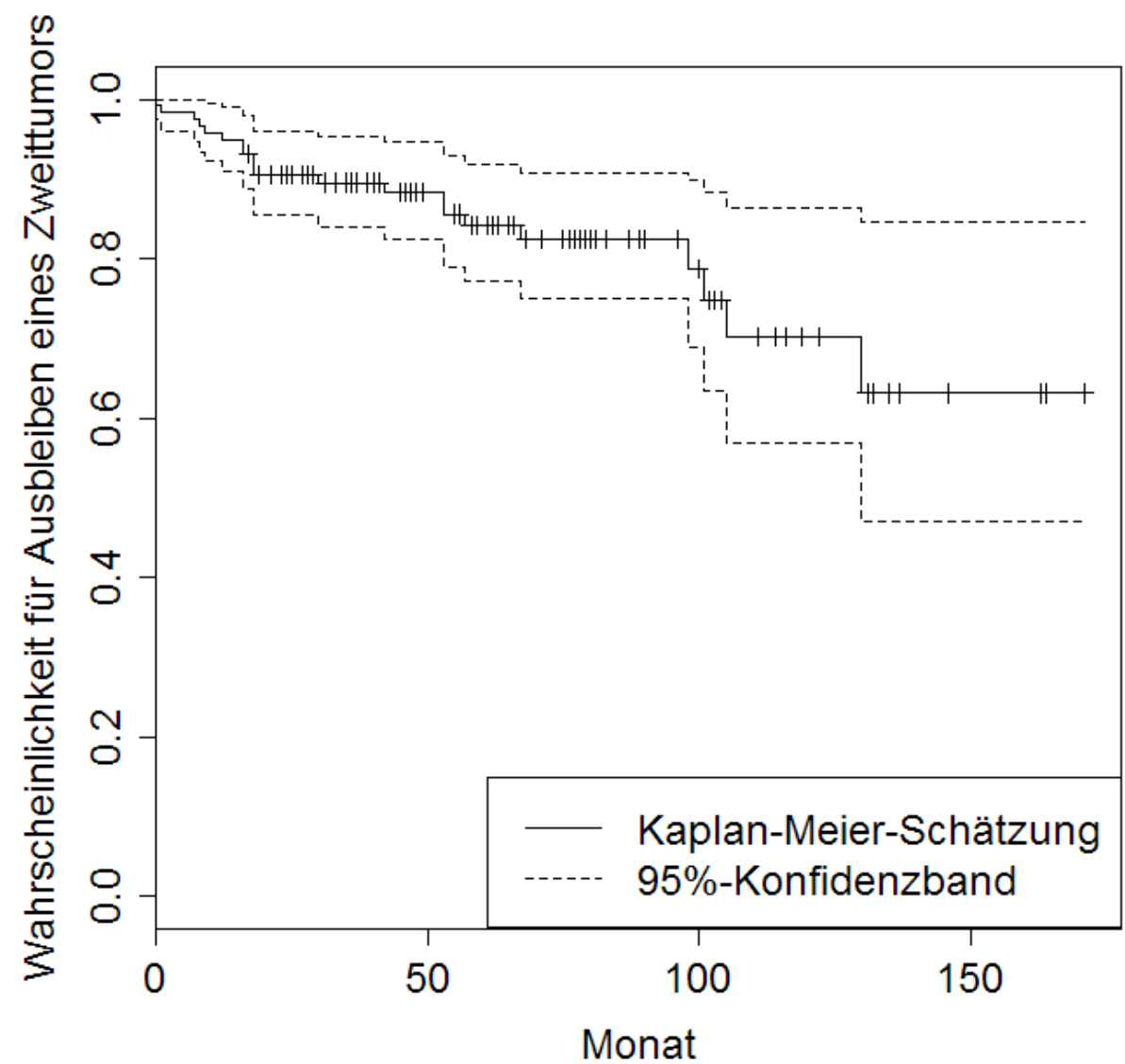

Abbildung 8: Darstellung der Gesamtwahrscheinlichkeit eines Zweittumorausbleibens anhand einer Kaplan-Meier-Kurve.

In einer anderen statistischen Auswertung wird beschrieben $\mathrm{zu}$ welchem Zeitpunkt die Häufigkeit des Auftretens eines Zweittumors am höchsten ist. Dabei wird deutlich, dass ein großer Teil der Zweittumoren, das bedeutet 11 der 21 diagnostizierten Zweittumore, innerhalb der ersten 20 Monate nach der Behandlung des Primärtumors auftritt (Abbildung 9). 


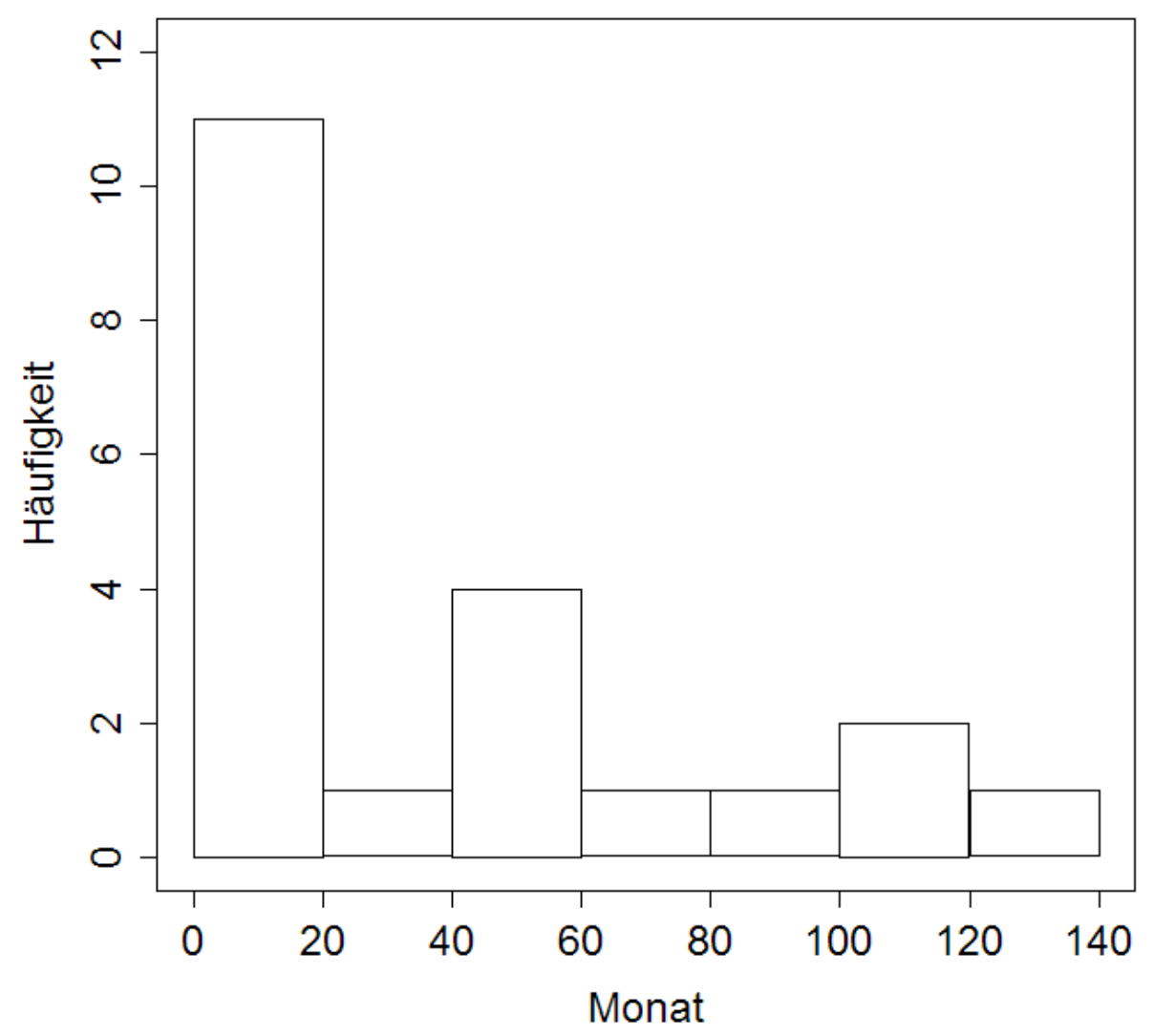

Abbildung 9: Darstellung der Häufigkeiten des Auftretens eines Zweittumors innerhalb eines bestimmten Zeitraums

Bei Betrachtung der Risikofaktoren Nikotin und Alkohol kann gezeigt werden, dass von den 21 Zweittumorpatienten nur 2 Patienten bis zum Zeitpunkt des Therapiebeginns des HNOTumors nicht geraucht haben. Alle anderen wiesen 30 bis 110 Packungsjahre auf.

Von den 2 Nichtraucher-Patienten hat ein Patient einen Zweittumor im HNO-Bereich und ist ehemaliger Alkoholiker und ein Patient ist mit ossären Metastasen auffällig geworden.

Die Analyse des Alkoholkonsums ergab bei 14 Patienten einen positiven Befund, während 8 Patienten einen ehemaligen oder aktuellen Alkoholabusus angaben. Weitere 7 Patienten verneinten allerdings den Alkoholgenuss komplett.

Die Patienten mit Zweittumoren im Ösophagus haben beide einen Alkoholabusus betrieben. Der dritte gastrointestinale Tumor, der in unserer Studie diagnostiziert wurde, war ein GIST. Außerdem konnte bei 4 Alkoholikern ein Zweittumor im HNO-Bereich nachgewiesen werden. Die anderen beiden alkoholabhängigen Patienten hatten ein Nierenzell- und ein Bronchialkarzinom. 
$\mathrm{Zu}$ beachten ist, dass die Analyse des Nikotin- und Alkoholkonsums ausreichend detailliert nur bis zum Zeitpunkt der Therapie des HNO-Tumors durchgeführt werden konnte.

Wie der Noxenkonsum im Verlauf nach Therapieabschluss aussah, konnte nicht ausreichend dokumentiert werden.

Tabelle 4: Nikotin- und Alkoholkonsum der Patienten

\begin{tabular}{|l|l|}
\hline Parameter & $\begin{array}{l}\text { Insgesamt } \\
\mathbf{n = 1 1 8}\end{array}$ \\
\hline $\begin{array}{l}\text { Nikotin } \\
\text { Ja }\end{array}$ & 102 \\
Nein & 16 \\
\hline Alkohol & 71 \\
Ja & 47 \\
Nein & \\
\hline
\end{tabular}


4. Diskussion

\subsection{Inzidenz von Zweittumoren und Screening der Luftwege}

Die vorliegende Arbeit beschäftigt sich mit der Inzidenz von Zweittumoren im Bereich der Luft- und Speisewege bei Patienten, welche zuvor aufgrund eines Hals-Nasen-Ohren-Tumors kurativ behandelt wurden. Hierfür wurden in dieser Arbeit bei insgesamt 118 Patienten im Rahmen der Routinenachsorge Daten von Untersuchungen (Computertomographie, ÖGD, Spiegelung des HNO-Bereiches) prospektiv analysiert. Die Ergebnisse zeigten dabei eine hohe Inzidenz von bislang unentdeckten und bis zum Untersuchungszeitpunkt klinisch asymptomatischen Tumoren. So wurden insgesamt 21 Tumore diagnostiziert und histologisch gesichert. Hiervon befanden sich 10 Zweittumore in der Lunge, 3 Zweittumore im Gastrointestinaltrakt, 7 Zweittumore im HNO-Bereich und 2 Zweittumore in anderen Lokalisationen. Ein Patient hatte zum Zeitpunkt der Untersuchungen sowohl ein Ösophaguskarzinom, als auch einen Zweittumor im HNO-Bereich.

Dabei wurden in der CT 16 von 27 Malignomverdachtsfällen bestätigt, während in der HNOUntersuchung 7 von 7 Malignomverdachtsfällen bestätigt wurden. Durch die ÖGD konnten insgesamt 3 von 4 Malignomverdachtsfällen gesichert werden.

Sowohl der Grundgedanke als auch die Ergebnisse der vorliegenden Arbeit zeigen sich dabei gut vergleichbar mit dem Studiendesign und Daten andere Studien. Besonders das rechtzeitige effektive Screening der Luftwege spielt in der weltweiten Literatur eine wichtige Rolle:

Eine Arbeitsgruppe von Henschke et al. gründete z.B. ein „Early Lung Cancer Action Project“, in dem von 1993 bis 19981.000 asymptomatische Patienten ein Basisscreening mit einer Low-Dose CT- Aufnahme des Thorax und einer konventionellen Röntgen-ThoraxAufnahme mit jährlichen Folgeaufnahmen erhielten. Voraussetzung zur Teilnahme war ein Alter von mindestens 60 Jahren, mindestens 10 Packungsjahren, kein vorangegangenes Tumorleiden und ein Allgemeinzustand, der eine thorakale Operation erlauben würde. 46\% der Patienten waren weiblich, 54\% männlich, das mittlere Alter lag bei 67 Jahren, die mittlere Packungsjahre-Anzahl bei 45 und eine positive Asbest-Anamnese lag bei 14\% vor. Die Auswertungen der CTs zeigten, verglichen mit einem konventionellen Röntgenbild des Thorax, dreimal mehr nicht-kalzifizierte Rundherde. Des Weiteren waren von den kleineren UICC-Stadium-I-Tumoren 83\%, also 19 von 23, zu diesem Zeitpunkt auf einem 
konventionellen Röntgenbild nicht sichtbar. Von den 27 detektierten Tumoren waren 96\% im weiteren Verlauf resektabel und $85 \%$ tatsächlich im UICC-Stadium I. Es erfolgte keine unnötige Thorakotomie aufgrund eines gutartigen Knotens. Aufgrund dieser Ergebnisse folgerten Henschke et al., dass ein Basis-CT-Screening sich gut für die frühere Diagnostik von Bronchialkarzinomen bei Risikopatienten mit Tabakvorgeschichte und einem Mindestalter von 60 Jahren eignet und es auf eine kosteneffiziente Weise mehr Möglichkeiten gibt, eine kurative Therapie zu erreichen (Henschke et al. 2001).

Swensen et al. führten 2005 in ihrer prospektiven Studie ein CT-Screening über 5 Jahre durch, bei dem jeder der 1520 Teilnehmer jährlich eine Aufnahme mit einem low-dose SpiralComputertomographen erhielt. Die Teilnehmer waren alle mindestens 50 Jahre alt und wiesen mehr als 20 Packungsjahre auf. Das Studienkollektiv bestand aus 52\% Männern und 48\% Frauen. 61\% waren aktuelle Raucher, 39\% ehemalige Raucher. Nach 5 Jahren wurden bei 1118, 74\% der Patienten, insgesamt 3356 nicht kalzifizierte Rundherde gefunden. Hierdurch wurden 68 Bronchialkarzinome bei 66 Teilnehmern diagnostiziert, was bedeutet, dass $4 \%$ von 1520 Teilnehmern und $2 \%$ von 3356 nicht kalzifizierten Rundherden Malignome waren. Die Inzidenz von Stadium-I-Nicht-kleinzelligen Bronchialkarzinomen lag bei 61\% (Swensen et al. 2005). Die daraus resultierenden Ergebnisse bezüglich eines Benefits werden im nächstfolgenden Kapitel 4.2 diskutiert.

Eine weitere Studie von Sone et al. untersuchte von 1996 bis 19985483 Personen japanischer Herkunft zwischen 40 und 74 Jahren mit jährlichen Spiral-CT-Aufnahmen mit einem lowdose-CT. Diese Personen bekamen schon über ein anderes Programm jährlich eine konventionelle Röntgen-Thorax-Aufnahme und eine Sputum-Untersuchung. Untersucht wurde, ob mit Spiral-CT-Screenings Bronchialkarzinome früher diagnostiziert werden können und ob diese dazu beitragen können, die Mortalitätsrate zu senken. Verglichen wurden die Probanden mit von Alter, Geschlecht und Nikotinkonsum passenden 2 Kontrollpersonen, die nur eine konventionelle Röntgen-Thorax-Aufnahme erhielten. Es wurde unterschieden zwischen Rauchern und Nicht-Rauchern und möglicherweise gutartigen, aber verdächtigen Läsionen, karzinomverdächtigen Läsionen und unklaren Rundherden. Nach Abschluss der 2 Jahre ergaben sich 19 Bronchialkarzinome, 14 Patienten mit Verdacht auf ein Bronchialkarzinom, 3 mit gutartigen, aber verdächtigen Läsionen und 2 Patienten mit unklaren Rundherden. 18 Tumore wurden mit einer Operation bestätigt, einer wurde klinisch diagnostiziert. Bei 4 Teilnehmern wurde der Befund auch in der konventionellen Aufnahme gesehen. Somit lag die Lungenkrebsdetektionsrate durch eine zusätzliche CT-Diagnostik mit $0,48 \%(0,52 \%$ bei Rauchern und $0,46 \%$ bei Nicht-Rauchern) signifikant höher als bei den 
Standardmethoden (0,03-0,05\%). Damit trafen Sone et al. die Aussage, dass CT-Aufnahmen aussagekräftiger sind und eine frühere Diagnostik ermöglichen (Sone et al. 1998).

\subsection{Mortalität und Prognose}

\subsubsection{Eigene Daten}

Insgesamt bleibt es in der hier vorliegenden Arbeit unklar, ob mit den durchgeführten Untersuchungen tatsächlich eine Mortalitätssenkung erreicht werden konnte. Um dazu eine Aussage treffen zu können, hätte man die untersuchten Patienten über einem längeren Zeitraum beobachten, Daten über Todeszeitpunkt und -Ursache eruieren, sowie Vergleiche mit anderen Patientenkollektiven anstellen müssen. Allerdings konnten von den 21 bestätigten Malignomen 18, also 85,7\%, in kurativer Absicht behandelt werden. Alle diese Patienten befanden sich in einem asymptomatischen und auch formell in einem früheren Tumorstadium, welches für einen kurativen Therapieansatz geeignet war. Eine mögliche Senkung der tumrobedingten Mortalität bei diesen Patienten kann somit nicht ausgeschlossen, aber auch nicht bewiesen werden.

\subsubsection{Andere Studienergebnisse}

An dieser Stelle ist besonders eine aktuelle Studie aus den USA vom „National Lung Screening Trial Research Team 2011“ zu nennen, welche sich mit der Mortalitätssenkung bei Lungenkrebs durch eine Computertomographie-Screeninguntersuchung beschäftigt. Hierbei wurden von 2002 bis 200453.454 Personen mit hohem Risiko, einen Lungentumor zu entwickeln, randomisiert aufgeteilt in 2 Gruppen. Hohes Risiko bedeutete Personen im Alter zwischen 55 und 74 Jahren mit einer Tabakvorgeschichte von mindestens 30 Packungsjahren. Ehemalige Raucher wurden eingeschlossen, wenn sie innerhalb der letzten 15 Jahre den Konsum eingestellt hatten. Ausgenommen wurden Personen, die vor kurzem die Diagnose eines Lungentumors erhalten haben, innerhalb der letzten 18 Monate eine CT-Untersuchung des Thorax erhalten haben, mit Hämoptysis aufgefallen sind oder einen ungeklärten Gewichtsverlust von mehr als 6,8 kg im letzten Jahr aufwiesen.

Die eine Gruppe erhielt jährlich über 3 Jahre ein Thorax-CT, die andere eine konventionelle Röntgen-Aufnahme des Brustkorbs. Die relative Reduktion der Mortalität aufgrund eines Lungentumors bei einem CT-Screening der Lunge betrug 20,0\% ( $p=0,004)$. Die Todesrate 
aufgrund jeglicher anderer Erkrankungen war bei der Gruppe, die die Computertomographie erhielt, im Vergleich zur Röntgen-Thorax-Gruppe um 6,7\% ( $\mathrm{p}=0,02)$ erniedrigt (Aberle et al. 2011a, Aberle et al. 2011b).

Swensen et al. beschäftigten sich ebenfalls mit der Frage einer folgenden Mortalitätssenkung, allerdings gab es in der bereits im vorherigen Kapitel beschriebenen Studie keinen konkomitanten Rückgang der späteren UICC-Stadien, sodass ein Vergleich mit dem „Majo Lung Cancer Project“, einer Studie aus den Siebzigern, die ein Lungenkrebs-Screening mit einer konventionellen Röntgen-Thorax-Aufnahme und einer Sputum-Untersuchung durchführte (Marcus et al. 2000) mit der Frage, ob es eine mehr als 50\% Veränderung in der Wahrscheinlichkeit der Detektion von Stadium I nicht-kleinzelligen Karzinomen gibt, kein signifikantes Ergebnis ergab. 48 Teilnehmer starben bis zum Abschluss der Studie, davon waren 9 Lungenkrebsassoziiert. Einer dieser Patienten starb postoperativ nach der Lungenoperation. Die Mortalitätsrate für Bronchialkarzinome betrug 1,6 pro 1000 pro Jahr.

Zusammengefasst sind Swensen et al. der Meinung, dass das CT-Screening bei Risikopatienten für Bronchialkarzinome die Diagnose von Tumoren in früheren Stadien ermöglicht. Auf diese Weise können Tumore in früheren Stadien im Vergleich zu konventionellen Röntgen-Thorax-Aufnahmen diagnostiziert werden. Ein eindeutiges Ergebnis, ob es dabei insgesamt zu einer Stadienveränderung bei Diagnosestellung kommt, konnte aber nicht beschrieben werden. Es bleibt also die Frage zu klären, ob folglich weniger Spätstadien diagnostiziert werden oder, ob nur eine Überdiagnostik erfolgt. Dies gilt insbesondere, da in dieser Studie ein sehr hoher Anteil falsch-positiver Befunde (Rate von 92,4-96,0\%) von gutartigen Rundherden erzeugt wurde, welcher im Gegensatz zu der Studie von Henschke et al. und den Ergebnissen der vorliegenden Studie häufig durch Operationen abgeklärt wurde, welche hohe Kosten, Mortalitätsrisiken und Lebensqualitätsverlust mit sich bringen können. Zusammenfassend, zeigte sich in dieser Studie kein Nutzen des CTScreenings von Risikopatienten bezüglich der Mortalität (Swensen et al. 2005).

Bei einem durchgeführten Follow-up der im vorherigen Kapitel bereits beschriebenen Arbeit von Sone et al. bis Juli 2006, was 2006 in „Lung Cancer“ ebenfalls von Sone et al. publiziert wurde, wurden für die 59 Patienten mit Bronchialkarzinom Überlebensraten angegeben. Insgesamt betrug die 5-Jahres-Überlebensrate 91,5\% und die 10-Jahres-Überlebensrate 86,2\% bezogen auf den Tod aufgrund eines Bronchialkarzinoms. Es ergaben sich signifikante Unterschiede in den Überlebensraten für das Stadium I und die Stadien II bis IV $(p=0,06)$, wobei die Überlebensrate für kleinere Stadien deutlich besser war. Bei Unterscheidung von Rauchern und Nicht-Rauchern ergab sich eine vergleichsweise schlechtere Prognose für 
Raucher mit Adenokarzinomen vom Mix-Typ und anderen soliden Befunden. Insgesamt bezeichnen Sone et al. es als Nachteil, dass sich in ihrem Programm sehr viele Nicht-Raucher mit gut-differenzierten Adenokarzinomen befanden, die eine bessere Prognose aufweisen. Insgesamt stellt sich allerdings in dieser Studie ein besseres 5-Jahres-Überleben mit CTScreenings dar (Sone et al. 2007).

In einer anderen Studie von Mahadevia et al. (2003) erhielten in einer Modellstudie mit Hilfe einer Computersimulation 100.000 hypothetische 60-jährige starke Raucher (mindestens 20 Packungsjahre), in gutem Allgemeinzustand, jährlich eine Spiral-CT-Bildgebung des Thorax. Sie wurden mit einer Gruppe verglichen, die keine CT-Untersuchungen erhielt. Das Kollektiv wurde nochmals in drei Untergruppen unterteilt, die entweder das Rauchen weiter fortsetzten, zum Zeitpunkt des Screenings mit dem Rauchen aufhörten oder schon seit 5 Jahren aufgehört hatten. Bei diesen Gruppen wurde ein Screening über 20 Jahre simuliert.

Um dies zu verwirklichen, wurden Daten und Ergebnisse von 4 verschiedenen publizierten großen Studien analysiert (Henschke et al. 1999, Henschke et al. 2001, Sone et al. 1998, Sone et al. 2001, Swensen et al. 2002, Sobue et al. 2002). Parameter wie Teilnehmereigenschaften (z.B. Alter oder Raucherstatus), Eigenschaften der verwendeten Screeningteste (Sensitvität und Spezifität), Eigenschaften der untersuchten Erkrankung, also des Bronchialkarzinoms (Behandlung, Prognose und Lebensqualität) und Eigenschaften des Screening-Programms selbst (Effektivität, statistische Fehler) wurden mit Hilfe der Studien und anderer Literatur, wie den Daten des „National Cancer Institute“ von 2001 und anderen berechnet (Jaklitsch et al. 1996, Sagel et al. 1978, Bach et al. 2001). Auf der Basis dieser Berechnungen wurde eine Basis-Analyse erstellt, die die Kosteneffektivität der Untersuchungen berechnet. Ebenfalls wurden zwei Sensitivitätsanalysen durchgeführt, von denen eine mit den Extremparametern für ein Screening berechnet wurde, die andere mit den gegenteiligen Extremen gegen ein Screening.

Gemessen wurden so die Vorteile des Screenings, indem der absolute und relative Unterschied des Todeszeitpunktes aufgrund des Lungentumors ermittelt wurde. Das Ergebnis zeigte eine Mortalitätsreduktion von 13\%. Mahadevia et al. legten den Schwerpunkt auf die Kosteneffizienz eines solchen Screenings. Am effizientesten war das Screening bei der Gruppe der kontinuierlichen Raucher bei Screeningstart zwischen 55 und 60 Jahren. Insgesamt ist das Screening nur sinnvoll bei Beginn vor dem Hauptrisikoalter für Bronchialkarzinome, das zwischen dem 67. und 72. Lebensjahr liegt.

In der Zusammenfassung kamen sie zu dem Ergebnis, dass das Screening von Bronchialkarzinomen mit CT-Aufnahmen in Anbetracht der niedrigen substanziellen 
Reduktion der Mortalitätsrate, den vielen falsch positiven Ergebnissen und vor allem den hohen Kosten nicht empfehlenswert ist (Mahadevia et al. 2003).

\subsubsection{Vergleich mit Screening-Untersuchungen anderer Tumorentitäten}

Besonders bei den häufigsten Tumorentitäten, wie dem Mammakarzinom bei Frauen und dem Prostatakarzinom bei Männern gibt es schon seit längerem Screeningverfahren, die zur regelmäßigen Anwendung kommen.

So haben in Deutschland alle Frauen zwischen 50 und 69 Jahren alle 2 Jahre ein Recht auf eine Mammographie. Eine der entscheidenden Arbeiten hierfür ist eine Studie aus Schweden von Tabár et al. über die Auswirkungen vom Mammographiescreening auf die Mortalität der am Mammakarzinom Erkrankten über 30 Jahre. Im Rahmen dieses Projekts wurden insgesamt 133.065 Frauen zwischen 40 und 74 Jahren in 2 verschiedenen schwedischen Bezirken in 2 Gruppen randomisiert.

Eine Gruppe erhielt im Abstand von 24-33 Monaten regelmäßig eine Mammographie, die andere Gruppe erhielt die übliche klinische Vorsorgeuntersuchung. Begonnen wurde 1977 bzw. 1978 und die Screeningphase dauerte ca. 7 Jahre. Das maximale Follow-up bestand aus 29 Jahren.

Das Ergebnis zeigte zwar eine relative Mortalitätssenkung von 30\%, in absoluten Zahlen konnte damit aber gezeigt werden, dass von 414 Frauen, nur ein Tod aufgrund eines Mammakarzinoms verhindert werden konnte.

Dies korrespondiert mit einem geretteten Leben pro 1334 durchgeführten Mammographien bzw. 1677, bezogen auf die Zahlen der unabhängigen „Swedish Cancer Society“.

Umgekehrt bedeutet dies, dass bei 1000 Frauen, die alle 2 Jahre im Alter von 40 bis 69 eine Mammographie erhalten, zwischen 8 und 11 Krebsassoziierte Todesfälle verhindert werden könnten.

Diese Studie zeigt wie unterschiedlich die Aussage von relativen und absoluten Zahlen sein kann. Eine Senkung der Mortalität um 30\% verspricht eine enorme Erfolgsrate, betrachtet man allerdings die „,number needed to treat“ von 414 Frauen, um einen brustkrebsassoziierten Todesfall zu verhindern, ist das Ergebnis schon weniger beeindruckend (Tabár et al. 2011).

Ein vergleichbares Screening beim Mann ist die Bestimmung des prostataspezifischen Antigens (PSA) im Blut zur Vorsorgeuntersuchung des Prostatakarzinoms. 
In einer Metaanalyse von Ilic et al. wurden 5 randomisiert kontrollierte Studien zur PSABestimmung und der daraus resultierenden Mortalitätssenkung des Prostatakarzinoms zusammengefasst. Insgesamt wurden dabei 341.351 Patienten eingeschlossen, deren Alter zwischen 50 und 74 Jahren lag. Die Dauer des Follow-ups betrug zwischen 7 und 15 Jahren.

Die Analyse aller 5 Studien zusammen zeigte keine signifikant erhöhte Reduktion der prostatakarzinomassoziierten Mortalität im Vergleich zu den Kontrollgruppen. Einzig und allein bei der größten aller Studien, die „European Randomized Study of Screening for Prostate Cancer", kurz ERSPC, konnte in einer Untergruppe von Männern zwischen 55 und 69 Jahren eine signifikante relative Reduktion der Mortalität von 20\% beschrieben werden. Eine Untergruppenanalyse aller Studien zeigte, dass die prostatakarzinomspezifische Mortalität nicht vom Alter abhängt, in dem die Teilnehmer untersucht wurden.

Allerdings war die Diagnose eines Prostatakarzinoms in den Screeninggruppen signifikant höher als in den Kontrollgruppen. Keine der Studien diskutierte allerdings die Problematik des Effektes des Screenings auf Lebensqualität der Patienten und dem hieraus entstehenden Kostenfaktor.

Außerdem fiel eine sehr hohe Rate von bis zu 75,9\% von falsch positiven Ergebnissen und einer Über-Diagnostik von bis $\mathrm{zu} 50 \%$ in der ERSPC-Studie auf. Ebenfalls wurden nachteilige Konsequenzen, wie die transrektale Ultraschalluntersuchung und die Risiken der Biospsien, wie Blutung oder Infektion, außen vor gelassen (Ilic et al. 2006).

In dieser Metaanalyse wird deutlich, dass auch etablierte Screeningverfahren nicht zwangsläufig zu einer Mortalitätssenkung führen und dass allgemeine Patientenkollektive oft zu heterogen sind, um eine sinnvolle Aussage zu treffen.

4.3 Zusammenfassung der vorliegenden Studienergebnisse bezüglich des Benefits eines Computertomographie-gestützten Screenings auf Lungentumore

Die Anzahl von Studien bezüglich des Benefits eines Computertomographie-gestützten Screenings auf Lungentumore ist enorm. Die Schlussfolgerungen aber sind sehr unterschiedlich. Die führenden Gründe gegen ein Screening stützen sich hauptsächlich auf den negativen Kosten-Nutzen-Vergleich, da eine regelmäßige Computertomographie, im Gegensatz zu einer konventionellen Röntgen-Thorax-Aufnahme, einen deutlich höheren Kostenfaktor mit sich bringt. Ebenfalls als negativ für die Patienten wird beschrieben, dass die falsch positiven Ergebnisse einen unnötigen Eingriff in die Körperintegrität hervorrufen. Im Extremfall bedeutet dies eine unnötige Thorakotomie mit allen bedenklichen Operations- 
und Narkoserisiken. In der Studie zu der hier vorliegenden Arbeit wurden 2 unnötige Bronchoskopien durchgeführt, bei denen sich der Tumorverdacht nicht bestätigen ließ und 2 operative Tumorresektion, die kein Malignom ergaben, sondern stattdessen ein Aspergillom und ein Epitheloidzellgranulom. Allerdings besteht zumindest beim Aspergillom die Therapie ebenfalls aus einer Resektion. Insgesamt kann man nicht davon sprechen durch diese Eingriffe, das Risiko für die Patienten unnötig erhöht bzw. ihre Lebensqualität gesenkt zu haben.

Bedeutsamer ist aber die rechtzeitige Erkennung der Tumore in einem Stadium, in dem eine kurative Behandlung möglich ist.

In den oben beschriebenen Studien postulieren die Autoren des National Lung Screening Trial, Henschke et al. und Sone et al., dass beim Screening mit Hilfe der Thorax-CT signifikant mehr Teilnehmer mit kleineren Stadien detektiert wurden und diese auch zumindest in der Follow-up-Studie von Sone et al. eine deutlich bessere Überlebenschance haben.

Auch in der hier vorliegenden Arbeit konnten von den 21 bestätigten Malignomen 18, also $85,7 \%$, kurativ behandelt werden. Die Patienten befanden sich also alle in einem asymptomatischen, entsprechend auch in einem früheren Stadium, welches für einen kurativen Therapieansatz geeignet war.

Allerdings wird auch deutlich, dass die meisten Arbeitsgruppen die Screeninguntersuchungen ausschließlich für bestimmte Risikogruppen für sinnvoll erachten. Dies bedeutet vornehmlich, dass nur Patienten mit Tabakanamnese und Erreichen eines bestimmten Alters einbezogen werden. Dies stellt eine ganz spezielle Patientengruppe dar, bei der diskutiert werden kann, ob ein regelhaftes Screening auf Lungentumore, aber auch Tumore im oberen Speiseweg sinnvoll und zu fordern ist.

\subsection{Betrachtung des speziellen Kollektivs dieser Arbeit}

Studien zur konventionellen Röntgen-Thorax-Untersuchung (Haas et al. 2001, Boysen et al. 1992) sind sich einig, keinen ausreichenden Benefit zu erreichen, außer möglicherweise eine psychologische Absicherung der Patienten, dass alles in Ordnung sei (Guerts et al. 2006). Betrachtet man die mittels Computertomographie gestützten Screenings auf Bronchialkarzinome, ist die Meinung weniger eindeutig, häufiger aber wird schlussgefolgert, dass es bei einer bestimmten Risikogruppe als sinnvoll erachtet wird. Hier zeigt sich der entscheidende Unterschied zu der hier vorliegenden Arbeit. Auch hier findet man zwar einen 
Altersmedian von 62 Jahren und auf 102 der 118 Patienten trifft eine positive Tabakanamnese zu. Allerdings wurden zusätzlich zu diesen Risikofaktoren alle 118 Teilnehmer zuvor kurativ an einem Tumor im Hals-Nasen-Ohren-Bereich behandelt.

Wenn man also ein spezielleres Risiko-Patientenkollektiv betrachtet, welches in der Vergangenheit bereits schon einmal an einem Tumor erkrankt ist, der von den gleichen Risikofaktoren hervorgerufen werden kann, ist ein möglicher Nutzen zumindest der CTScreeninguntersuchungen zu postulieren. Eine HNO-Untersuchung ist bis zu 5 Jahre nach dem Primärtumor ohnehin obligat. Über eine ÖGD-Untersuchung kann aufgrund der vorliegenden Ergebnisse diskutiert werden. Zur Erhärtung der hier vorliegenden orientierenden Ergebnisse wäre eine multizentrische prospektiv randomisierte Studie notwendig, welche diese Daten weiter überprüft.

Es gibt bereits Literatur, die sich mit dem Risiko von der Entstehung von Zweittumoren bei Patienten mit Hals-Nasen-Ohren-Tumoren beschäftigt:

In einer Studie von León et al. im Mai 1999 wurde bereits retrospektiv bei 1845 Patienten, die von 1984 bis 1995 aufgrund eines Tumors im Hals-Nasen-Ohren-Bereich behandelt wurden, das Auftreten von Zweittumoren, deren Charakteristika und die Auswirkungen auf das Überleben der Teilnehmer untersucht. Im Ergebnis entwickelten 16\% einen Zweittumor, wobei das jährliche Entstehungsrisiko konstant bei $4 \%$ lag. $40 \%$ der Zweittumore befanden sich ebenfalls im HNO-Bereich, $31 \%$ in der Lunge und $9 \%$ im Ösophagus. Somit befanden sich $80 \%$ der Zweittumore im aerodigestiven Trakt und 20\% außerhalb dieser Körperregionen. Insgesamt zeigte sich, dass das Risiko einen Zweittumor zu entwickeln besonders hoch bei Patienten mit positiver Tabakanamnese ist, wohingegen Patienten mit einem Primärtumor im Larynxbereich ein niedrigeres Risiko aufwiesen. Raucher hatten ein 1,6- bis 1,7-fach höheres Risiko im Vergleich zu Nicht-Rauchern. Durch das Auftreten eines Zweittumors verschlechterte sich die Überlebensrate signifikant $(\mathrm{p}<0,001)$ (León et al. 1999).

Im Jahr 2010 erweiterte León et al. in einer weiteren Arbeit ihre Aussage soweit, dass Patienten mit einem HNO-Primärtumor nicht nur ein signifikant erhöhtes Risiko haben, einen Zweittumor zu entwickeln, sondern auch einen Dritt- und Vierttumor. Demnach wurde eine retrospektive Studie mit klinischen Daten von 3631 prospektiv gesammelten Patientendaten durchgeführt, die aufgrund eines HNO-Tumors behandelt wurden. Hier wurde nicht nur das Risiko für die Entwicklung eines Zweittumors untersucht, sondern es wurden auch Risiken für Dritt- und Vierttumore analysiert. Bei der Evaluation stellte sich heraus, dass das Risiko kontinuierlich anstieg. Das jährliche Risiko einen Zweittumor zu entwickeln lag bei 3,8\%, 
dieses Risiko scheint konstant zu bleiben. Das Risiko einen Dritttumor zu entwickeln liegt aber bereits bei 5,1\%. Noch größer ist das Risiko für einen Vierttumor, was bei 7,8\% liegt. Damit ließen sich signifikante Unterschiede im Risiko der Entwicklung eines Zweit-, Drittoder Vierttumors darstellen.

In der Zusammenfassung postulierten die Autoren ein ansteigendes Risiko für Patienten mit vorherigen HNO-Tumoren, im Verlauf weitere Tumore zu entwickeln (Léon et al. 2010).

Auch bei dieser Arbeit konnte eine erhöhte Inzidenz von Zweittumoren festgestellt werden. Bei 17,8\% der untersuchten Teilnehmer konnte ein zweites Malignom nach einem Primärtumor im HNO-Bereich gefunden wurden.

Da aus vorangegangener Literatur bereits der Schwerpunkt auf den Aerodigestivtrakt gelegt wurde, wurde der Fokus auch in dieser Studie auf diese Organsysteme gelegt. Wie auch in der Studie von León et al. befand sich der Großteil der Zweittumore mit 47,6\% in der Lunge, gefolgt von Zweittumoren im HNO-Bereich selbst (33,3\%). Es stellt sich als sinnvoll heraus, sich bei einem Zweittumorscreening auf diejenigen Hauptrisikoorgane zu beschränken, die für die Entwicklung eines Malignoms identische Risikofaktoren aufweisen. Im oben beschriebenen Teilnehmerprofil zeigt sich, dass 86\% Raucher waren und $60 \%$ als alkoholkrank bezeichnet werden mussten.

Von den 21 Zweittumorpatienten in dem hier untersuchten Studienkollektiv haben nur 2 Patienten nicht geraucht, alle anderen wiesen 30 bis 110 Packungsjahre auf. Die Analyse des Alkoholkonsums ergab bei 14 Tumorpatienten einen positiven Befund.

Zusammenfassend sollte man bei der Wahl der Patienten, die sich regelmäßigen Vorsorgeuntersuchungen unterziehen sollten, eine Anamnese dieser Risikofaktoren mit einbeziehen.

Es wird ebenfalls in zwei Arbeiten diskutiert, dass die Tumore einer Achse folgen. Entweder der respiratorischen Achse oder der digestiven Achse, was bedeutet, dass Bronchialkarzinome eher Zweittumore nach Larynxtumoren sind, wobei ösophageale Tumore meist nach Hypopharynxkarzinomen entstehen (León et al. 1999, Bradley und Bradley 2010). Diese Tatsache konnte in unserer Studie nicht bestätigt werden. Allerdings kann in der vorliegenden Arbeit nicht auf eine vergleichbar große Teilnehmerzahl zurückgegriffen werden. Ebenfalls kann die Aussage, ob Patienten mit Larynxkarzinomen seltener betroffen sind, wie in der Studie von León et al. von 1999 postuliert, nicht bestätigt werden.

In einer anderen Arbeit von Di Martino et al. von 2002 wird eine Aussage über den Zeitpunkt des Auftretens der Zweittumore getroffen. Dieser beläuft sich angeblich zu $40 \%$ auf nach der regulären 5-Jahres-Nachsorge (Di Martino et al. 2002). Ebenso beschrieben Bradley und 
Bradley 2010, dass die meisten metachronen Zweittumore 4 Jahre nach dem Primärtumor auftreten (Bradley und Bradley 2010). Diese Aussagen ließen sich in unserem Teilnehmerklientel nicht bestätigen. 10 der 21 detektierten Zweittumore traten bei dieser Arbeit in den ersten 20 Monaten nach Behandlung des Ersttumors auf. Das hier beschriebene Kollektiv muss allerdings als relativ inhomogen gewertet werden, da die meisten Primärtumore zum Zeitpunkt der Untersuchung noch nicht außerhalb der 5-Jahres-Nachsorge lagen. Dies wäre in weiteren Arbeiten mit größeren Teilnehmerzahlen und Zeitspannen zu untersuchen.

\subsection{Screening-Untersuchungen bei HNO-Tumorpatienten}

Im Gegensatz zu den allgemeinen Lungenkrebs- Vorsorgestudien, die sich sehr intensiv mit dem Benefit eines frühen Screenings beschäftigen, beschreiben die meisten Artikel zu Zweittumoren bei HNO-Patienten hauptsächlich das absolute Risiko der Patienten, an einem Zweittumor zu erkranken. Eine detaillierte Analyse sinnvoller Screeninguntersuchungen existiert aber bislang nicht. Dies erklärt möglicherweise auch, weshalb es kein einheitliches Schema in der Nachsorge von HNO-Tumor-Patienten gibt.

Allein die Autoren Bradley und Bradley geben in einer Arbeit von 2010 einen Vorschlag für ein ideales Überwachungsprotokoll zur Identifikation von Zweittumoren bei HNOTumorpatienten. Hier spielt die PET-CT eine wichtige Rolle, denn diese soll, zumindest bei von ihnen bezeichneten „Hoch-Risiko-Patienten“, vor Behandlung des Primärtumors, 3-4 Monate nach Behandlung, 12 Monate nach Behandlung, 2 Jahre und 3 Jahre nach Behandlung durchgeführt werden und dann abwechselnd mit einer Endoskopie von HNO, Lunge und Ösophagus mit Autofluoreszenz jährlich wiederholt werden. Bei sogenannten ,intermediär oder Niedrig-Risiko-Patienten“" wird eine jährliche Trippelfluoreszenzendoskopie und eine PET-CT für insgesamt 3 Jahre empfohlen (Bradley und Bradley 2010).

Leider ist dieses Vorgehen zum heutigen Stand zumindest in Deutschland unrealistisch, denn diese Techniken überschreiten deutlich das Kostenlimit eines regelmäßigen Screenings. Es müssen also kostengünstigere Alternativen gesucht werden.

Außerdem sind insbesondere die Daten zu Vorsorgeuntersuchungen auf ösophageale Zweittumore bei HNO-Tumor-Patienten wenig zahlreich und wenig vielversprechend.

Eine Arbeitsgruppe aus Frankreich hat bei 1560 Patienten mit primärem HNO-Tumor zweimal jährlich über 10 Jahre eine Ösophago-Gastro-Duodenoskopie durchgeführt. Bei 3,2\% wurden metachrone ösophageale Zweittumore entdeckt, die sich in einer mittleren Zeit 
von 47 Monaten entwickelten. Auch hier wird postuliert, dass Patienten mit Tumoren im Oropharynx ein signifikant höheres Risiko für Zweittumoren in der gastrointestinalen Achse haben. Allerdings sind über die Hälfte der Patienten nicht an dem detektierten Zweittumor verstorben und das mittlere Überleben betrug nur 16 Monate, sodass in dieser Arbeit der Benefit eines systematischen Screenings auf ösophageale Zweittumore in Frage gestellt wird (Petit et al. 2001).

Eine weitere asiatische Studie analysierte die Daten des nationalen Krebsregisters Taiwans nach Patienten mit initialem Tumor in Mundhöhle und Pharynx und ösophagealen Zweittumoren bei diesen. Die Analyse lief über 25 Jahre und schloss 33.787 Patienten ein.

Hier konnte eine Aussage über Patientengruppen mit höheren Risiken gegenüber solchen mit niedrigeren getroffen werden. Zum Beispiel Patienten mit Hypopharynxkarzinomen oder Patienten, die die Diagnose des Ersttumors im Alter $\leq 50$ Jahre erhielten. Allerdings betrug auch hier die Gesamtinzidenz eines Ösophagustumors nur 0,59\%, sodass auch hier das positive Kosten-Nutzen-Verhältnis in Frage gestellt wurde und der Schwerpunkt auf die Detektion bestimmter Hoch-Risiko-Gruppen gelegt wurde (Lee et al. 2009).

Auch in der hier vorliegenden Arbeit sind die Ergebnisse zum Nutzen einer regelmäßigen ÖGD diskussionswürdig. Insgesamt wurden nur 3 gastrointestinale Tumore festgestellt, davon war einer ein GIST, der nicht in die gemeinsame Risikogruppe gezählt werden kann. Allerdings konnte bei den anderen beiden Plattenepithelkarzinomen des Ösophagus eine kurative Therapiestrategie mit Tumorresektion verfolgt werden.

Andererseits spielt der Kostenfaktor bei einer regelmäßigen ÖGD eine untergeordnete Rolle gegenüber einer CT-Untersuchung. So gab ein großer Teil der Patienten im vorherigen Aufklärungsgespräch Refluxbeschwerden an und es wurden bei knapp 20\% Schleimhautentzündungen befundet und mit Protonenpumpenhemmern behandelt.

Dies kann man im weiteren Sinne auch zu einer effektiven Vorsorge zählen, da diese Befunde potentiell ebenfalls zu einer malignen Entartung der Ösophagus- oder Magenschleimhaut führen können.

\subsection{Ausblick und Problematik}

In der vorliegenden Arbeit wurde eine spezielle Untergruppe analysiert, die im Vergleich zu anderen Gruppen ein deutlich erhöhtes Zweittumorrisiko aufweist.

Allerdings konnte mit dieser Arbeit keine abschließende Aussage über eine tatsächliche Senkung der Mortalität getroffen werden. Hierfür sind ein langes Follow-up und eine deutlich 
größere Teilnehmerzahl, sowie Vergleiche mit anderen Patientenkollektiven notwendig. Ohne eine sichere Aussage über eine Verlängerung des Überlebens machen zu können, ist es fragwürdig, ein solches Screening durchzuführen.

Die Tumore, die hierbei erkannt werden, sind zum größten Teil symptomlos, die Patienten fühlen sich im weitesten Sinne gesund. Wird nun aufgrund der Screening-Untersuchung ein Zweitmalignom diagnostiziert, bedeutet dies in der Regel eine erneute notwendige Therapie. Dies bedeutet für den Patienten meist zunächst eine Lebensqualitätverschlechterung aufgrund von Operationen, Chemotherapien oder Bestrahlungen sowie langen Krankenhausaufenthalten. Dieser Lebenseinschnitt mit Beeinträchtigung der Lebensqualität und der Allgemeinzustandsverschlechterung muss vor der Festlegung einer eindeutigen Therapieempfehlung zu der tatsächlich möglichen Lebensverlängerung abgewogen werden. Möglicherweise kann aber eine Standardisierung eines Screeningprogramms für sogenannte „Hoch-Risiko-Patienten“ im Sinne von Patienten mit ausgeprägten Risikofaktoren und vorausgegangenen Primärtumoren im HNO-Bereich die Mortalität aufgrund eines nicht früh genug erkannten Zweittumors senken, ohne die Lebensqualität deutlich zu verschlechtern. 


\section{Zusammenfassung}

Durch eine Vielzahl von Studien und Literaturnachweisen ist bekannt, dass Patienten mit den Risikofaktoren Nikotin- und Alkoholabusus ein deutlich erhöhtes Risiko aufweisen, an malignen Tumoren zu erkranken. Dies führt zu der Überlegung, bei diesen Patienten mittels Screeningverfahren die Früherkennung von Tumoren zu verbessern. Dies gilt insbesondere auch für Patienten, welche zuvor bereits kurativ aufgrund eines Tumors im HNO-Bereich behandelt worden sind, da die heutige multimodale Therapie immer bessere Überlebensraten ermöglicht und gerade diese Patienten häufig entsprechende Risikofaktoren aufweisen. Somit werden insbesondere bei diesen Patienten im weiteren Verlauf nach kurativer Behandlung des Kopf-Hals-Tumors zunehmend Zweittumore in der Lunge oder dem Ösophagus lebenslimitierend. Ein Screeningverfahren ist allerdings hauptsächlich dann als sinnvoll zu bewerten, wenn für die Patienten hierdurch auch ein Überlebensvorteil entsteht.

In der vorhandenen Literatur über die Früherkennung von Tumoren im Bereich der Lunge oder des Ösophagus wurden bislang fast ausschließlich Patienten mit den bekannten Risikofaktoren untersucht, detaillierte Analysen speziell für HNO-Tumorpatienten fehlen aber weitestgehend. In der vorliegenden Arbeit konnte gezeigt werden, dass durch Früherkennungsverfahren, wie eine Computertomographie des Thorax und eine ÖsophagoGastro-Duodenoskopie bei ebendiesen HNO-Tumor-Patienten Zweittumore zu einem frühen Zeitpunkt erkannt werden können. So konnte insbesondere durch die Computertomographie eine hohe Zahl an bislang klinisch unauffälligen Zweittumoren entdeckt werden.

Zwar kann durch diese Arbeit noch keine entgültige Aussage über eine mögliche Lebensverlängerung durch die in den Screeninguntersuchungen gefundenen Tumore getroffen werden, eine sehr wichtige Erkenntnis ist aber, dass 18 der 21 Zweittumore mit einem erneuten kurativen Therapieansatz behandelt werden konnten, da die Tumorstadien überwiegend weder lokal fortgeschritten noch fernmetastasiert waren.

Um eine abschließende Aussage über mögliche langfristige Überlebensvorteile und die tatsächliche Anzahl erkannter Zweittumore zu gewinnen, müssen die gewonnenen Ergebnisse zukünftig an einem größeren Patientenkollektiv im Rahmen einer prospektiven multizentrischen Studie validiert werden. 
6. Tabellen

Tabelle 5: T-Stadien der Tumoren von Lippe, Mundhöhle und Oropharynx

\begin{tabular}{|c|l|}
\hline TX & Primärtumor kann nicht beurteilt werden \\
\hline T0 & Kein Anhalt für einen Primärtumor \\
\hline Tis & Carcinoma in situ \\
\hline T1 & $\begin{array}{l}\text { Tumorgröße liegt bei maximal } 2 \mathrm{~cm} \text { in seiner } \\
\text { größten Ausdehnung }\end{array}$ \\
\hline T2 & $\begin{array}{l}\text { Tumorgröße liegt bei mindestens 2 cm aber } \\
\text { nicht bei mehr als 4 cm }\end{array}$ \\
\hline T3 & Tumorgröße mit mehr als 4 cm Ausdehnung \\
\hline T4 & $\begin{array}{l}\text { Tumor mit Ausdehnung auf } \\
\text { Nachbarstrukturen (Tiefeninfiltration) }\end{array}$ \\
\hline & \multicolumn{1}{|c}{} \\
\hline
\end{tabular}

(Wittekind et al. 2005, S.16-18)

Tabelle 6: T-Stadien der Tumoren des Hypopharynx

\begin{tabular}{|c|l|}
\hline T1 & $\begin{array}{l}\text { Tumor beschränkt sich auf einen Unterbezirk } \\
\text { (Sin. piriformis, Postkrikoidregion, } \\
\text { Hypopharynxhinterwand) und < 2cm }\end{array}$ \\
\hline T2 & $\begin{array}{l}\text { Tumor ausgedehnt auf mehrere Bezirke oder } \\
\text { Nachbarbezirk ohne Fixation an die } \\
\text { Umgebung und }>2 \mathrm{~cm} \text { aber }<4 \mathrm{~cm}\end{array}$ \\
\hline T3 & $\begin{array}{l}\text { Tumor ausgedehnt auf mehrere Bezirke oder } \\
\text { Nachbarbezirke mit Fixation an die } \\
\text { Umgebung (Larynx, oberer Ösophagus, } \\
\text { Oropharynx) oder > 4cm }\end{array}$ \\
\hline T4 & $\begin{array}{l}\text { Tumor mit Überschreiten des Hypopharynx } \\
\text { und massivem Tumoreinbruch in die } \\
\text { Nachbarstrukturen (Larynx, Halsweichteile } \\
\text { und andere Nachbarorgane) }\end{array}$ \\
\hline
\end{tabular}

(Wittekind et al. 2005, S.31-34) 
Tabelle 7: T-Stadien der Tumoren der Glottis

\begin{tabular}{|c|l|}
\hline T1 & $\begin{array}{l}\text { Tumor ist auf die Stimmlippe begrenzt, mit } \\
\text { normaler Beweglichkeit } \\
\text { Tumor ist auf eine Stimmlippe begrenzt }\end{array}$ \\
\hline T1a & Tumor befällt beide Stimmlippen \\
\hline T1b & $\begin{array}{l}\text { Tumor breitet sich auf einen oder beide } \\
\text { Nachbarbezirke aus und schränt die } \\
\text { Stimmlippenbeweglichkeit ein }\end{array}$ \\
\hline T2 & $\begin{array}{l}\text { Tumor auf den Larynx begrenzt, die } \\
\text { Stimmlippen sind fixiert und/oder Invasion } \\
\text { des Postkrikoidbezirkes, präepiglottischen } \\
\text { Gewebes, paraglottischen Raumes }\end{array}$ \\
\hline T4a und T4b & $\begin{array}{l}\text { stimmen mit der Einteilung des } \\
\text { supraglottischen Karzinoms überein. }\end{array}$ \\
\hline
\end{tabular}

(Wittekind et al. 2005, S.45-47)

Tabelle 8: T-Stadien der Tumoren der Supraglottis

\begin{tabular}{|c|l|}
\hline T1 & $\begin{array}{l}\text { Tumor ist auf einen Unterbezirk der } \\
\text { Supraglottis beschränt und die Stimmlippen } \\
\text { sind frei beweglich }\end{array}$ \\
\hline T2 & $\begin{array}{l}\text { Tumor infiltriert mehr einen und einen } \\
\text { weiteren benachbarten Bereich der } \\
\text { Supraglottis bzw.: außerhalb, ohne dabei die } \\
\text { Stimmlippen zu beeinträchtigen }\end{array}$ \\
\hline T3 & $\begin{array}{l}\text { Tumor ist auf den Larynx begrenzt mit } \\
\text { Stimmlippenfixation und/oder infiltriert den } \\
\text { Postkrikoidbezirk oder präepiglottisches } \\
\text { Gewebe. }\end{array}$ \\
\hline T4a & $\begin{array}{l}\text { Tumor infiltriert durch Schildknorpel } \\
\text { und/oder breitet sich außerhalb des Larynx } \\
\text { aus }\end{array}$ \\
\hline T4b & $\begin{array}{l}\text { Tumor infiltriert den Prävertebralraum, } \\
\text { mediastinale Strukturen oder umschließt die } \\
\text { A.carotis interna }\end{array}$ \\
\hline &
\end{tabular}

(Wittekind et al. 2005, S.41-44) 
Tabelle 9: T-Stadium der Tumoren der Subglottis

\begin{tabular}{|c|l|}
\hline T1 & Tumor auf Subglottis begrenzt \\
\hline T2 & $\begin{array}{l}\text { Tumor breitet sich auf Stimmlippen aus mit } \\
\text { oder ohne Bewegungseinschränkung }\end{array}$ \\
\hline T3 & $\begin{array}{l}\text { Tumor auf den Larynx begrenzt mit } \\
\text { Stimmlippenfixation }\end{array}$ \\
\hline T4a und b & $\begin{array}{l}\text { sind wie beim supraglottischen Karzinom } \\
\text { definiert }\end{array}$ \\
\hline
\end{tabular}

(Wittekind et al. 2005, S.48-49)

Tabelle 10: Stadieneinteilung der Lymphknotenmetastasen bei HNO-Tumoren

\begin{tabular}{|c|l|}
\hline $\mathrm{N} 0$ & Keine regionäre Lymphknotenmetastasen \\
\hline $\mathrm{N} 1$ & $\begin{array}{l}\text { in einem ipsilateralen Lymphkonten mit einer } \\
\text { maximalen Größe von 3 cm }\end{array}$ \\
\hline $\mathrm{N} 2 \mathrm{a}$ & $\begin{array}{l}\text { Metastase in einem ipsilateralen } \\
\text { Lymphknoten mit einer Größe zwischen 3 bis } \\
6 \mathrm{~cm}\end{array}$ \\
\hline $\mathrm{N} 2 \mathrm{~b}$ & $\begin{array}{l}\text { Metastasen in multiplen ipsilateralen } \\
\text { Lympknoten mit einer maximalen Größe von } \\
6 \text { cm }\end{array}$ \\
\hline $\mathrm{N} 2 \mathrm{c}$ & $\begin{array}{l}\text { Metastasen in bilateralen oder kontralateralen } \\
\text { Lymphknoten mit einer maximalen Größe } \\
\text { von 6 cm }\end{array}$ \\
\hline $\mathrm{N} 3$ & $\begin{array}{l}\text { Metastasen mit einer Größe von mehr als 6 } \\
\text { cm }\end{array}$ \\
\hline
\end{tabular}

(Wittekind et al. 2005, S.6-12)

Tabelle 11: Stadieneinteilung der Fernmetastasen bei HNO-Tumoren

\begin{tabular}{|l|l|}
\hline M0 & Keine Fernmetastasen \\
\hline M1 & Fernmetastasen \\
\hline
\end{tabular}

(Wittekind und Meyer 2010, S.27) 
Tabelle 12: UICC-Stadien

\begin{tabular}{|c|c|c|c|}
\hline Stadium 0 & Tis & N0 & M0 \\
\hline Stadium I a & T1 & N0 & M0 \\
\hline Stadium I b & T2 & N0 & M0 \\
\hline Stadium II a & T3 & N0 & M0 \\
\hline Stadium II b & T4 & N0 & M0 \\
\hline Stadium III a & Jedes T & N1 & M0 \\
\hline Stadium III b & Jedes T & N2 & M1 \\
\hline Stadium IV & Jedes T & Jedes N & \\
\hline
\end{tabular}

(Sinn et al. 2003) 


\section{Literaturverzeichnis}

Aberle D, Adams A, Berg C, Black W, Clapp J, Fagerstrom R, Gareen I, Gatsonis C, Marcus P, Sicks J (2011a):

The national lung screening trial: Overview and study design

Radiology 258, 243-53

Aberle D, Adams A, Berg C, Black W, Clapp J, Fagerstrom R, Gareen I, Gatsonis C, Marcus P, Sicks J (2011b):

Reduced lung-cancer mortality with low-dose computed tomographic screening

N Engl J Med 365, 395-409

Bach PB, Cramer LD, Schrag D, Downey RJ, Gelfand SE, Begg CB (2001):

The influence of hospital volume on survival after resection for lung cancer

N Engl J Med $\underline{345}, 181-8$

Barwad A, Sood S, Gupta N, Rajwanshi A, Panda N, Srinivasan R (2011):

Human papilloma virus associated head and neck cancer: a PCR based study

Diagn Cytopathol doi:10.1002/dc.21667

Bernier J (2008):

A multidisciplinary approach to squamous cell carcinomas of the head and neck: an update Curr Opin Oncol 20, 249-55

Bernier J, Domenge C, Ozsahin M, Matuszewska K, Lefèbvre JL, Greiner RH, Giralt J, Maingon P, Rolland F, Bolla M (2004):

Postoperative irradiation with or without concomitant chemotherapy for locally advanced head and neck cancer

N Engl J Med $\underline{350}, 1945-52$

Boenninghaus HG und Lenarz T:

HNO, 12.Auflage;

Springer Verlag, Heidelberg 2005

Bollschweiler E, Leers J, Hölscher H (2009):

Epidemiologie des Ösophagus- und Magenkarzinoms

Der Gastroenterologe $\underline{4}, 193-201$

Boysen M, Lovdal O, Tausjo J, Winther F (1992):

The value of follow-up in patients treated for squamous cell carcinoma of the head and neck Eur J Cancer $\underline{28}$, 426-430

Bradley PJ, Bradley PT (2010):

Searching for metachronous tumours in patients with head and neck cancer: the ideal protocol!

Curr Opin Otolaryngol Head Neck Surg $\underline{18}, 124-33$ 
Cao S, Liu Z, Jia W, Huang Q, Liu Q, Guo X, Huang T, Ye W, Hong M (2011):

Fluctuations of epstein-barr virus serological antibodies and risk for nasopharyngeal carcinoma: a prospective screening study with a 20 -year follow-up

PLoS One $\underline{6}$, e19100

Chin D, Boyle GM, Porceddu S, Theile DR, Parsons PG, Coman WB (2006):

Head and neck cancer: past, present and future

Expert Rev Anticancer Ther $\underline{6}, 1111-8$

Cooper JS, Pajak TF, Forastiere AA, Jacobs J, Campbell BH (2004):

Postoperative concurrent radiotherapy and chemotherapy for high-risk squamous-cell carcinoma of the head and neck

N Engl J Med $\underline{350}$, 1937-44

Curado MP, Hashibe M (2009):

Recent changes in the epidemiology of head and neck cancer

Curr Opin Oncol 21, 194-200

Dhooge IJ, De Vos M, Van Cauwenberge PB (1998):

Multiple primary, malignant tumors in patients with head and neck cancer: results of a prospective study and future perspectives

Laryngoscope $\underline{108}, 250-6$

Di Martino E, Sellhaus B, Hausmann R, Minkenberg R, Lohmann M, Esthofen MW (2002):

Survival in second primary malignancies of patients with head and neck cancer

J Laryngol Otol $\underline{116}, 831-8$

Franceschi S, Talamini R, Barra S, Barón AE, Negri E, Bidoli E, Serraino D, La Vecchia C (1990):

Smoking and drinking in relation to cancers of the oral cavity, pharynx, larynx, and esophagus in northern Italy

Cancer Res $\underline{50}$, 6502-7

Franzen A:

Hals-Nasen-Ohrenheilkunde, 3. Auflage;

Urban \& Fischer Verlag, München 2007

Gao Y, Hu N, Ding T, Giffen C, Goldstein AM, Taylor PR (2011):

Risk factors for esophageal and gastric cancers in Shanxi Province, China: a case control study

Cancer Epidemiol 35, e91-9

Guerts TW, Ackerstaff AH, Van Zandwijk N, Hart AA, Hilgers FJ, Balm AJ (2006):

The psychological impact of annual chest X-ray follow-up in head and neck cancer

Acta Otolaryngol 126, 1315-1320

Haas I, Hauser U, Ganzer U (2001):

The dilemma of follow-up in head and neck cancer patients

Eur Arch Otorhinolaryngol 258, 177-183 
Henschke CI, McCauley DI, Yankelevitz DF, Naidich DP, McGuiness G, Miettinen OS, Libby DM, Pasmantier MW, Koizumi J, Altorki NK (1999):

Early lung cancer action project: overall design and findings from baseline screening Lancet $\underline{354}, 99-105$

Henschke CI, McCauley DI, Yankelevitz DF, Naidich DP, McGuiness G, Miettinen OS, Libby D, Pasmantier M, Koizumi J, Altorki N (2001):

Early lung cancer action project: a summary of the findings on baseline screening

The Oncologist $\underline{6}, 147-152$

Hentschel S, Katalinic A:

Das Manual der epidemiologischen Krebsregistrierung

Zuckschwerdt Verlag, München 2008

Herth F, Becker HD (2001):

New aspects in early detection and local staging of early lung cancer

Lung Cancer 34, 7-11

Ilic D, O’Connor D, Green S, Wilt T (2006):

Screening for prostate cancer

Cochrane Database Syst Rev $\underline{3}$, CD004720

Jaklitsch MT, DeCamp MM Jr, Liptay MJ, Harpole DH Jr, Swanson SJ, Mentzer SJ, Sugarbaker DJ (1996):

Video-assisted thoracic surgery in the elderly: a review of 307 cases

Chest $\underline{110}, 751-8$

Lee KD, Lu CH, Chen PT, Chan C, Lin JT, Huang CE, Chen CC, Chen MC (2009):

The incidence and risk of developing a second primary esophageal cancer in patients with oral and pharyngeal carcinoma: a population-based study in Taiwan over a 25 year period BMC Cancer $\underline{9}, 373$

León X, Quer M, Santiago D, Orús C, Lopez-Pousa A, Burgués J (1999):

Second neoplasm in patients with head and neck cancer

Head Neck 21, 204-210

Léon X, Martinez V, Lopez M, Garcia J, Quer M (2010):

Risk of third and fourth tumors in patients with head and neck cancer

Head Neck $\underline{32}$, 1467-72

MacMahon H, Austin JH, Gamsu G, Herold CJ, Jett JR, Naidich DP, Patz EF Jr, Swensen SJ, Fleischner Society (2005):

Guidelines for management of small pulmonary nodules detected on CT scans: a statement from the Fleischner Society

Radiology 237, 395-400

Mahadevia P, Fleisher L, Frick K, Eng J, Goodman S, Powe N (2003):

Lung cancer screening with helical computed tomography in older adult smokers: a decision and cost-effectiveness analysis

JAMA $\underline{289}, 313-322$ 
Marcus PM, Bergstralh EJ, Fagerstrom RM, Williams DE, Fontana R, Taylor WF, Prorok PC (2000):

Lung cancer mortality in the Majo Lung Project: impact of extended follow up

J Natl Cancer Inst 92, 1308-1316

Mashberg A, Samit A (1995):

Early Diagnosis of Asymptomatic Oral and Oropharyngeal Squamous Cancers

CA Cancer J Clin $\underline{45}, 328-51$

Mashberg A, Garfinkel L, Harris S (1981):

Alcohol as a primary risk factor in oral squamous carcinoma

CA Cancer J Clin $\underline{31}, 146-55$

Merletti F, Bofetta P, Ciccone G, Mashberg A, Terracini B (1989):

Role of tobacco and alcoholic beverages in the etiology of cancer of the oral cavity/oropharynx in Torino, Italy

Cancer Res $\underline{49}, 4919-24$

Moral M, Paramio JM (2008):

Akt pathway as a target for therapeutic intervention in HNSCC

Histol Histopathol 23, 1269-78

Morita M, Kumashiro R, Kubo N, Nakashima Y, Yoshida R, Yoshinaga K, Saeki H, Emi Y, Kakeji Y, Sakaguchi Y (2010):

Alcohol drinking, cigarette smoking, and the development of squamous cell carcinoma of the esophagus: epidemiology, clinical findings, and prevention

Int J Clin Oncol 15, 126-134

Morris LG, Sikora AG, Hayes RB, Patel SG, Ganly I (2011):

Anatomic sites and elevated risk of second primary cancer after an index head and neck cancer

Cancer Causes Control 22, 671-9

Offner FA (1997):

Ätiologie, Molekularbiologie und Pathologie des Plattenepithelkarzinoms des Oesophagus, Der Onkologe $\underline{3}, 620-8$

Pelucchi C, Tramacere I, Boffetta P, Negri E, La Vecchia C (2011):

Alcohol consumption and cancer risk

Nutr Cancer $\underline{63}$, 983-90

Petit T, Georges C, Jung G, Borel C, Bronner G, Flesch H, Massard G, Velten M, Haegele P, Schraub S (2001):

Systematic esophageal endoscopy screening in patients previously treated for head and neck squamous-cell carcinoma

Ann Oncol 12, 643-646

Pfister D, Ang K, Brizel D, Burtness B, Cmelak A, Colevas A, Dunphy F, Eilsele D, Gilbert J, Gillison M (2011):

Head and neck cancers

J Natl Compr Canc Netw $\underline{9}, 596-650$ 
Renz-Polster H, Krautzig S, Braun J

Basislehrbuch Innere Medizin, 4.Auflage

Urban \& Fischer Verlag, München 2008

Riede UN, Werner M, Schaefer HE:

Allgemeine und spezielle Pathologie, 5. Auflage;

Thieme Verlag, Stuttgart 2004

Sagel SS, Ferguson TB, Forrest JV, Roper CL, Weldon CS, Clark RE (1978):

Percutaneous transthoracic aspiration needle biopsy

Ann Thorac Surg 26, 399-405

Schweisfurth H, Kurbjuhn H (2004):

Epidemiologie und Ätiologie des Lungenkarzinoms

Brandenburgisches Ärzteblatt $\underline{4}, 126-127$

Simon C, Plinkert PK (2008):

Combined modality approaches in the treatment of head and neck cancer patients

HNO $\underline{56}, 575-84$

Sinn HP, Hermanek P, Wagner G, Wittekind CH (2003):

Organspezifische Tumordokumentation, 3.Auflage;

Internetfassung, Heidelberg 2003

Slaughter DP, Southwick HW, Smejkal W (1953):

Field cancerization in oral stratified squamous epithelium; clinical implications of multicentric origin

Cancer $\underline{6}, 963-8$

Sobue T, Moriyama N, Kaneko M, Kusumoto M, Kobayashi T, Tsuchiya R, Kakinuma R, Ohmatsu H, Nagai K, Nishiyama H (2002):

Screening for lung cancer with low-dose helical computed tomography: anti-lung cancer association project

J Clin Oncol 20, 911-20

Sone S, Takashima S, Feng L, Yang Z, Honda T, Maruyama Y, Hasegawa M, Yamanda T, Kubo K, Hanamura K (1998):

Mass screening for lung cancer with mobile spiral computed tomography scanner

The Lancet $\underline{351}, 1242-45$

Sone S, Nakayama T, Honda T, Tsushima K, Li F, Haniuda M, Takahashi Y, Suzuki T, Yamanda T, Kondo R (2007):

Long-term follow-up study of a population-based 1996-1998 mass screening programme for lung cancer using mobile low-dose spiral computed tomography

Lung Cancer 58, 329-341

St John MA, Abemayor E, Wong DT (2006):

Recent new approaches to the treatment of head and neck cancer

Anticancer Drugs 17, 365-75 
Strutz J, Mann W:

Praxis der HNO-Heilkunde, Kopf- und Halschirurgie

Thieme Verlag, Stuttgart-New York 2001

Swensen SJ, Jett JR, Sloan JA, Midthun DE, Hartman TE, Sykes AM, Aughenbaugh GL, Zink FE, Hillman SL, Noetzel GR (2002):

Screening for lung cancer with low-dose spiral computed tomography

Am J Respir Crit Care Med 165, 508-513

Swensen SJ, Jett JR, Hartman TE, Midthun DE, Mandrekar SJ, Hillman SL, Sykes AM, Aughenbaugh GL, Bungum AO, Allen KL (2005):

CT screening for lung cancer: five-year prospective experience

Radiology 235, 295-65

Tabár L, Vitak B, Chen T, Yen A, Cohen A, Tot T, Chiu S, Chen S, Fann J, Rosell J (2011):

Swedish two-county trial: impact of mammographic screening on breast cancer mortality during 3 decades

Radiology 260, 658-63

Tepperman BS, Fitzpatrick PJ (1981):

Second respiratory and upper digestive tract cancers after oral cancer

Lancet $\underline{1981,2}, 547-9$

Tsou YA, Hua CH, Tseng HC, Lin MH, Tsai MH (2007):

Survival study and treatment strategy for second primary malignancies in patients with head and neck squamous cell carcinoma and nasopharyngeal carcinoma

Acta Otolaryngol 127, 651-7

Watanabe A, Hosokawa M, Taniquchi M, TsujieH, Sasaki S (2007):

Head and Neck Cancer associated with esophageal cancer

Auris Nasus Larynx 눈, 207-11

Weiderpass E, (2010):

Lifestyle and cancer risk

J Prev Med Public Health $\underline{43}$, 459-471

Wittekind $\mathrm{CH}$, Meyer HJ:

TNM, 7.Auflage;

WILEY-VCH Verlag GmbH \& Co. KGaA, Weinheim 2010

Wittekind C, Klimpfinger M, Sobin LH:

TNM-Atlas, 5.Auflage;

Springer Medizin Verlag, Heidelberg 2005 
8. Danksagung

Herrn Prof. Dr. Dr. med. C. F. Hess danke ich für die Möglichkeit, diese klinische Arbeit in seiner Abteilung durchführen zu können.

Ich danke Herrn Prof. Dr. med. H. Christiansen, der die Durchführung der vorliegenden Studie ermöglicht und mich als mein Doktorvater sehr unterstützt hat.

Mein besonderer Dank gilt Herrn Dr. med. Hendrik Wolff, der durch unermüdliche Mitarbeit und Geduld zu jeder Zeit und besonders durch konstruktive Kritik, aber auch durch seinen nie endenden Optimismus maßgeblich zum Gelingen dieser Arbeit beigetragen hat.

Weiterhin möchte ich Herrn Prof. Dr. med. R. Rödel danken, welcher mir die Daten seiner Patienten zu Verfügung gestellt hat und die HNO-Untersuchungen durchgeführt hat.

Ebenfalls möchte ich Herrn Prof. Dr. med. Engelke und Frau Prof. Dr. med. MüllerDornieden danken, die sich mit der Befundung der Computertomographien beschäftigten und die Ösophago-Gastroskopien durchführten. 


\section{Lebenslauf}

Am 15. Januar 1985 wurde ich, Cornelia Ruth Marie Wolff, geborene Vienken, als Tochter von Dr. med. Rolf Richard Ludwig Vienken und seiner Ehefrau Brigitte Elisabeth Katharina Verbeek-Vienken als Erstes von zwei Kindern in Trier geboren.

Von 1991 bis 1995 besuchte ich die Keune Grundschule in Trier. Darauf folgend besuchte ich von 1995 bis 2004 das Friedrich-Wilhelm-Gymnasium in Trier, wo ich das Zeugnis der Allgemeinen Hochschulreife erlangte.

Im Wintersemester 2004 begann ich das Studium der Biologie an der Johannes-GutenbergUniversität in Mainz bis einschließlich des Wintersemesters 2005.

Im Sommersemester 2006 begann ich dann mein Studium der Humanmedizin an der Georg-August-Universität in Göttingen. Nach dem 4. vorklinischen Semester absolvierte ich im Wintersemester 2007/2008 den ersten Abschnitt der ärztlichen Prüfung.

Das Praktische Jahr absolvierte ich an der medizinischen Fakultät in Montpellier an den Krankenhäusern Saint-Eloi und Lapeyronie (Chirurgie), dem Helios Albert-SchweitzerKlinikum in Northeim (Innere Medizin) und der Universitätsmedizin in Göttingen mit dem Wahlfach Dermatologie.

Im Juli 2008 begann ich meine Dissertation in der Abteilung Strahlentherapie und Radioonkologie des Universitätsklinikums Göttingen. 